\title{
Performance of Predictive Indoor mmWave Networks with Dynamic Blockers
}

\author{
Andrea Bonfante, Lorenzo Galati Giordano, Irene Macaluso and Nicola Marchetti
}

\begin{abstract}
In this paper, we consider millimeter Wave (mmWave) technology to provide reliable wireless network service within factories where links may experience rapid and temporary fluctuations of the received signal power due to dynamic blockers, such as humans and robots, moving in the environment. We propose a novel beam recovery procedure that leverages Machine Learning (ML) tools to predict the starting and finishing of blockage events. This erases the delay introduced by current 5G New Radio (5G-NR) procedures when switching to an alternative serving base station and beam, and then reestablish the primary connection after the blocker has moved away. Firstly, we generate synthetic data using a detailed systemlevel simulator that integrates the most recent 3GPP 3D Indoor channel models and the geometric blockage Model-B. Then, we use the generated data to train offline a set of beam-specific Deep Neural Network (DNN) models that provide predictions about the beams' blockage states. Finally, we deploy the DNN models online into the system-level simulator to evaluate the benefits of the proposed solution. Our prediction-based beam recovery procedure guarantee higher signal level stability and up to $82 \%$ data rate improvement with respect detection-based methods when blockers move at speed of $2 \mathrm{~m} / \mathrm{s}$.
\end{abstract}

Index Terms-Millimeter-wave, 5G New Radio, dynamic blockage, machine learning, deep neural network.

\section{INTRODUCTION}

$\mathbf{T}$ HE integration of Machine Learning (ML) tools with wireless communication systems is envisioned as one of the essential steps towards future intelligent Radio Access Networks (RANs) [1], [2]. ML tools can be employed to design novel resource management and control methods to optimise the performance of 5-th Generation (5G) and beyond wireless systems [3]. One of the key technologies for $5 \mathrm{G}$ networks is millimeter Wave (mmWave) communication, where the large available bandwidth at mmWave frequencies can support extremely high capacity wireless links, offering data rates in the order of gigabits per second [4]-[6]. MmWave communications are well-suited for indoor network deployments [7], e.g. open offices, shopping centres and industrial spaces. Adopting multi-antenna arrays with analog-only or hybrid architectures enables forming narrow beams that provide high directivity gains and compensate for the severe pathloss [8]. Nevertheless, in this scenario, the mmWave links experience rapid and temporary fluctuations of the received signal power when they

A. Bonfante, I. Macaluso and N. Marchetti are with CONNECT Centre, Trinity College Dublin, Ireland (e-mail: bonfanta@tcd.ie; nicola.marchetti@tcd.ie; MACALUSI@tcd.ie). L. Galati Giordano is with Nokia Bell Labs, Stuttgart, Germany (e-mails: lorenzo.galati_giordano@nokia-bell-labs.com). This work was supported by Irish Research Council and by Nokia Ireland Ltd under Grant Number EPSPG/2016/106. encounter blocking objects, such as humans and robots moving in the environment [9]. The links' data rate may become highly intermittent, making it highly challenging to guarantee high data rate and low latency services [10], [11].

In general, the blockage effect follows non-periodic time dynamics and cannot be predicted in advance from the received power using time series forecasting models, as there is no indication that the blockage will happen until the blockage causes a significant change in the received signal power. Therefore, conventional beam recovery methods are based on blockage detection. For instance, the beam failure recovery (BFR) procedure adopted by Third Generation Partnership Project (3GPP) New Radio (NR) standard [12] and other methods shown in [13], [14] rely on a signal threshold, detecting the blockage events when the received signal power drops below a given value. The beam recovery operations yield a delay as they establish the backup beam after detecting the blockage, leading to a data rate loss every time the blocker intersects the link.

On the other hand, context data collected, for example, by using other mmWave links [15], [16], utilising the Sub-6 GHz spectrum bands [17], [18] or by adopting external sensors (e.g. camera [19], [20] and radar [21]), provide relevant information about the environment surrounding the mmWave link's path. Leveraging these data enables to predict if a blockage event will occur at any given time on the mmWave link. Thus, the blockage predictions can trigger the beam recovery operations ahead of the blockage events to avoid the data rate loss experienced by the detection methods. The early works [15][17], [19], [20] showed promising results when applying ML tools to the blockage prediction problem. Nevertheless, their analysis is limited to the single-User Equipment (UE) scenario, not accounting for multiple UEs, which would be a more realistic scenario for modelling mmWave network deployments and enable exploring the feasibility of applying ML predictions at the network level.

\section{A. Related Works}

In this section, we review state-of-the-art approaches that use ML tools to make the blockage prediction of mmWave links. There are three major techniques regarding the data acquisition for training the ML models: (i) using data acquired from the mmWave channel measurements, (ii) utilising complementary data from the Sub- $6 \mathrm{GHz}$ channel bands and (iii) collecting data through a camera or radar mounted on top of the mmWave Base Station (mmWave-BS). 
The authors of [15] consider using multi-link mmWave channel measurements as input data for a Long Short-Term Memory (LSTM) model that predicts the received signal power variations of one of the link in advance. In the single-cell scenario, signal-to-noise ratio (SNR) measurements related to both Line-of-Sight (LoS) and Non Line-of-Sight (NLoS) mmWave links with the serving Base Station (BS) need to be collected, requiring sweeping the beam towards the NLoS paths directions and interrupting the ongoing data communication with the UE. Moreover, in the multi-cell scenario, the method entails acquiring the SNR measurements from the LoS paths with neighbour BSs. In this case, the neighbour BSs perform beam sweeping, changing the beams towards UEs that are not served, and provide the additional SNR measurements. Thus, integrating the LSTM-based method proposed in [15] into the 5G NR mmWave network operations can be challenging to realise in practice and is still an open research problem. Differently in [16], the authors propose to use unsupervised online learning to predict the mmWave link state. This approach utilises the Multipath Components (MPC) of the mmWave channel as input data for the ML model; however, the predictions are limited to one blocker and rely on its location information, which is usually not available unless, for instance, the blocker is equipped with a localisation device. Another interesting work that relies on data acquired from the mmWave channel measurements is [22], where a UE moving in a vehicle utilises the ML model predictions to select the BS that takes over the communication to avoid the blockage. However, this work is suited for vehicular networks, and we consider this scenario outside the scope of this paper.

Another line of work focuses on using the Sub- $6 \mathrm{GHz}$ spectrum bands measurements to observe the rapid variations of the mmWave channel due to the blockage, tens of ms ahead of the blockage event [23]. Following this approach, in [17], the authors propose a Deep Neural Network (DNN) model that uses the Sub- $6 \mathrm{GHz}$ channel measurements to predict the beam state and the optimal beam of the codebook that maximises the achievable rate. Their results show a $90 \%$ probability of correct predictions of the mmWave link blockage state. This method is verified with the single-UE scenario, and it is impractical for the standalone (SA) mmWave network deployment.

Alternatively, in [19], the authors propose a ML-based predictor of received signal power that uses visual data acquired by a camera pointing to the BS-UE path. Similarly, in [20], the authors use visual data to predict the BS to which handover the connection. Conversely, the RadMAC project is a proof-ofconcept that deploys a mmWave radar on top of the mmWave BS [21]. RadMAC tracks with the radar the presence of moving objects, e.g., humans, that can eventually intersect the mmWave link, switching the beam preemptively to a backup link during the blockage events. The applicability of the visual and radar-based systems is proved for the single-UE case, where it is possible to cover the beam path serving the UE with a wide camera angle or with the radar system. However, practical mmWave systems use beam steering with codebookbased transmission to cover multiple UEs in the sector, making it more challenging to employ visual and radar-based methods to determine the blockage state for each beam.

\section{B. Contributions}

In this paper, we propose a novel beam recovery method that is integrated with DNN models to predict blockage events. This method relies on the early indication of the predictions to switch to an alternative beam pair, slightly before the blockage starts to impact the received signal power. Unlike the works [15]-[17], [19], [20], we consider extending the blockage prediction method to a more realistic multi-UE setting, enabling us to deploy the ML models into the network to study the data rate performance. Moreover, most of the existing works necessitate extra Hardware (HW) components, e.g. camera and Sub-6 GHz transceiver, while we rely exclusively on the mmWave channel measurements aggregated from multiple BSs. The ML model proposed in [15] uses input data that necessitate additional SNR measurements from the ones already defined in the 5G NR specifications. Instead, we use standard beam-quality measurements reported by the UEs to the BSs according to the 3GPP NR specification [24].

We consider a mmWave Indoor network with high UEs density and multiple BSs. We account for BSs employing multi-antenna arrays with analog beamforming architecture and codebook-based signal transmission. We incorporate into the 3GPP-based system-level simulator a geometric blockage model (Blockage model B) that ensures consistent results over time, space and frequency components of the channel [25]. The beams intersecting the blocker in locations that are spatially close have beam-quality measurements that follow similar temporal dynamics. Hence, the blocker presence at time $t$ on one beam can indicate the blockage presence on other beams in successive instants of time. Thus, we train a DNN model to predict the beam-specific blockage state, taking as input data the other beams' measurements exchanged with the neighbour BSs through a central controller. Our contributions can be summarised as follows:

1) We train a set of beam-specific DNN models with synthetic data generated through a 3GPP-based systemlevel simulator. The models exclusively use existing beam-quality measurements reported by the UEs to the BSs as indicated in the $5 \mathrm{G} \mathrm{NR}$ specifications.

2) We propose a novel multi-UE prediction-based method for beam recovery that uses the DNN models' output to control and initiate the beam switching in advance and complete this operation by the time the mmWave link becomes blocked.

3) We validate the prediction-based method by deploying the DNN models online into a 3GPP-based system-level simulator to verify the close match to the performance of an ideal method that has perfect knowledge of the future beam states. We then compare the prediction method to other two cases: $i$ ) adopting the beam recovery method based on detection and $i$ ) utilising a fixed beam method. We show that the prediction method switches to a backup beam earlier than the blockage event, and 


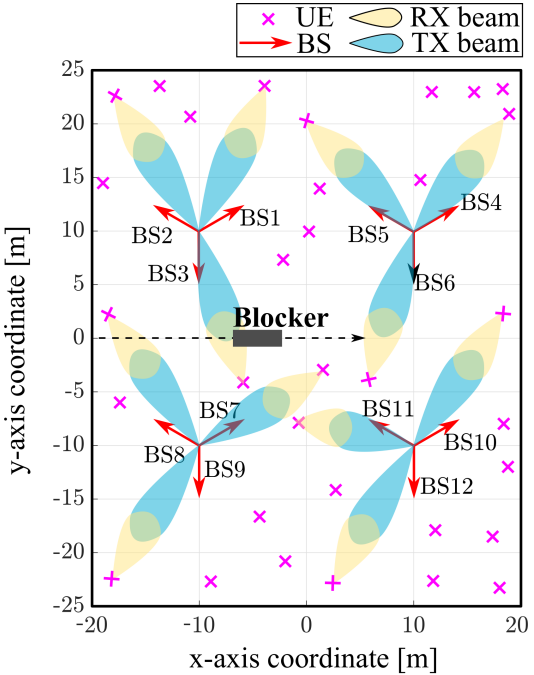

Fig. 1: Layout of the indoor mmWave network.

avoids the data rate loss that occurs with the detection method due to the delay in switching to the backup beam and without the switching for the fixed beam method.

4) We provide quantitative data rate results of the three methods varying the blocker speed. During blocked time instants, the prediction-based method improves the 25-th percentile of the fixed-beam and detection-based methods data rates by $238 \%$ and $24 \%$ for blocker speed of 1 $\mathrm{m} / \mathrm{s}$, and by $223 \%$ and $82 \%$ for blocker speed of $2 \mathrm{~m} / \mathrm{s}$. This shows the benefits of using the prediction-based method for the beam recovery operation, especially for the worst served UEs and higher blocker speed.

The remainder of this paper is organised as follows. Section II describes the system model and the mmWave Downlink (DL) transmission; Section IIII introduces the beam recovery method based on detection and the beam recovery method based on predictions; Section IV describes the procedure to obtain the beam state predictions; Section $\mathrm{V}$ presents the evaluation of the methods under analysis and the main results, and VI summarises the key findings and directions for future research. The following notation is used throughout the paper: boldface lower case and boldface upper case are used for column vectors $\mathbf{x}$ and matrices $\mathbf{X}$, respectively. $\mathbf{x}^{\top}$ denote the transpose of $\mathbf{x}$. A complex Gaussian random variable $x$ is denoted $x \sim \mathcal{C N}\left(\mu, \sigma^{2}\right)$, where $\mu$ is the mean and $\sigma^{2}$ is the variance. $[X]$ denotes the Iverson bracket that is defined to be 1 when $X$ is true and 0 when $X$ is false.

\section{System ModeL}

\section{A. Network Layout}

We consider the network layout depicted in Fig. 1 and inspired by the 3GPP Indoor Hot-spot scenario [25], [26], formed by four cell sites deployed on a rectangular grid with an Inter Site Distance (ISD) of 20 meters. Each cell site has three sectors, served respectively by three BSs oriented with an angle $\theta_{j}$ with $j \in\{1,2,3\}$ and operating at mmWave carrier

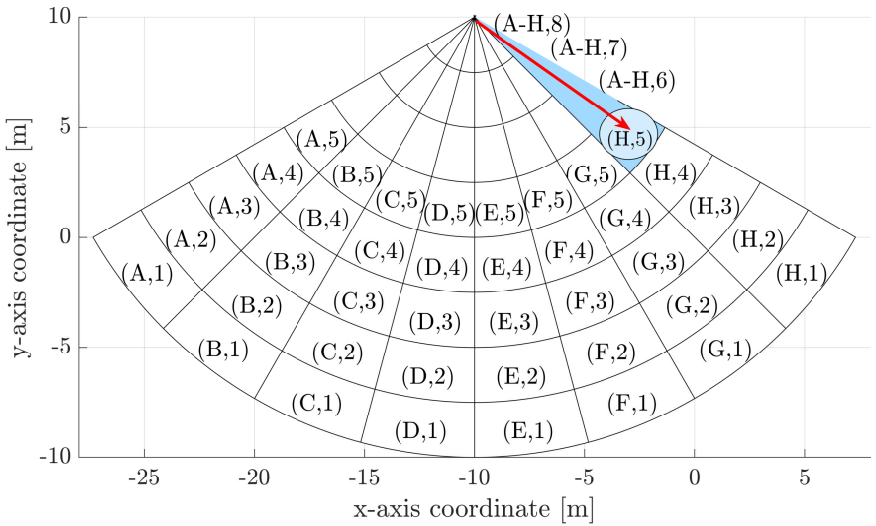

(a) Top-down view

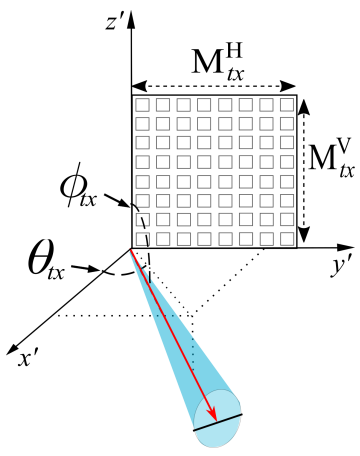

(b) Structure of the Tx UPA

Fig. 2: (a) Top-down view of the area in the $x-y$ plane illuminated by the Tx beams of the BS-3. (b) Structure of the UPA formed by $M_{T x}^{\mathrm{H}} \times M_{T x}^{\mathrm{V}}$ antennas and placed in the local coordinates system $\left(x^{\prime}, y^{\prime}, z^{\prime}\right)$. The Tx beam has azimuth $\theta_{T x}$ defined between the $\mathrm{x}^{\prime}$ axis and the Tx beam projection on the $\mathrm{x}^{\prime}-\mathrm{y}$ ' plane and elevation $\phi_{T x}$ defined between the $\mathrm{z}^{\prime}$ axis and the Tx beam direction.

frequency $f_{c}$, with bandwidth $B W$. The BSs in the mmWave network form the set $\mathcal{J}$ with cardinality $J$. We assume a set of $\mathcal{K}$ UEs uniformly distributed over a rectangular area of 50 meters by 40 meters and we consider dynamic blockers having dimension $w \times h$ moving at speed $v$ in the environment.

Assuming DL transmission, both the BS Transmitter (Tx) and the UE Receiver (Rx) employ Uniform Planar Array (UPA) structures with $M_{T x}=M_{T x}^{\mathrm{V}} \times M_{T x}^{\mathrm{H}}$ and $M_{R x}=$ $M_{R x}^{\mathrm{V}} \times M_{R x}^{\mathrm{H}}$ antennas spaced half-wavelength, where $M_{T x}^{\mathrm{V}}$ and $M_{R x}^{\mathrm{V}}$ are the number of $\mathrm{Tx}$ and $\mathrm{Rx}$ antennas in the vertical direction, and $M_{T x}^{\mathrm{H}}$ and $M_{R x}^{\mathrm{H}}$ are the ones in the horizontal direction. We assume the BS and UE implement analog beamforming with a single Radio Frequency (RF) chain, selecting beams from a pre-defined codebook. We denote as $\left(\theta_{T x}, \phi_{T x}\right)$ the azimuth and elevation Angle of Departures (AoDs) and as $\left(\theta_{R x}, \phi_{R x}\right)$ the azimuth and elevation Angle of Arrivals (AoAs) of the $\mathrm{Tx}$ and Rx beamforming vectors, respectively. The Tx beamforming vector for UPA can be expressed as $\mathbf{b}_{T x}\left(\theta_{T x}, \phi_{T x}\right)=$ $\frac{1}{\sqrt{M_{T x}^{\mathrm{H}} M_{T x}^{\mathrm{V}}}}\left[1, \ldots, e^{-j \pi\left(\left(M_{T x}^{\mathrm{H}}-1\right) \Theta_{T x}+\left(M_{T x}^{\mathrm{V}}-1\right) \Phi_{T x}\right)}\right]^{\top} \quad$ where $\Theta_{T x}=\sin \phi_{T x} \sin \theta_{T x}$ and $\Phi_{T x}=\cos \phi_{T x}$. Similarly, the 
Rx beamforming vector can be given as $\mathbf{b}_{R x}\left(\theta_{R x}, \phi_{R x}\right)=$ $\frac{1}{\sqrt{M_{R x}^{\mathrm{H}} M_{R x}^{\mathrm{V}}}}\left[1, \ldots, e^{\left.-j \pi\left(\left(M_{R x}^{\mathrm{H}}-1\right) \Theta_{R x}\right)+\left(M_{R x}^{\mathrm{V}}-1\right) \Phi_{R x}\right)}\right]^{\top}$ where $\Theta_{R x}=\sin \phi_{R x} \sin \theta_{R x}$ and $\Phi_{R x}=\cos \phi_{R x}$. The beamforming vectors $\mathbf{b}_{T x}$ and $\mathbf{b}_{R x}$ are identified with two beam IDs $l$ and $q$ defined as $l=\left\{1, \ldots, N_{\mathrm{CB}, T x}\right\}$ and $q=$ $\left\{1, \ldots, N_{\mathrm{CB}, R x}\right\}$, where $N_{\mathrm{CB}, T x}$ and $N_{\mathrm{CB}, R x}$ denote the cardinalities of Tx and Rx codebooks $\mathcal{B}_{T x}$ and $\mathcal{B}_{R x}$, respectively. Fig. 2a shows the top-down view of the area illuminated by the Tx beams of the BS-3. Each Tx beam of the codebook is steered towards the direction with $\operatorname{AoD}\left(\theta_{T x}, \phi_{T x}\right)$ as shown in Fig. 2b, and illuminates a specific area that is marked with a letter-number combination.

\section{B. Downlink Data Transmission}

Consider the DL of a NR-based system for mmWave cellular communications. The NR standard numerology employs a physical time-frequency resource division corresponding to 14 Orthogonal Frequency Division Multiplexing (OFDM) symbols for one Transmission Time Interval (TTI) in the time domain and 12 consecutive subcarriers in the frequency domain forming a Resource Block (RB) [27]. The system operates with the Time Division Duplex (TDD) scheme, and each BS uses beam sweeping to time-multiplex multiple UEs, serving one UE at a time. At a given time instant $t$, the DL signal transmitted from the BS $j$ to the UE $k$ can be expressed as

$$
y_{j, k}(t)=\sqrt{P_{b}} s_{k}(t) \mathbf{b}_{R x}^{\top} \mathbf{H}_{j, k}(t) \mathbf{b}_{T x}+\mathbf{b}_{R x}^{\top} z_{k}(t),
$$

where $P_{b}$ is the total power of the BS, $s_{k}(t)$ with $\mathbb{E}\left[\left|s_{k}(t)\right|^{2}\right]=$ 1 is the signal transmitted, $\mathbf{H}_{j, k}(t)$ represents the impulse response of the three dimensional (3D) Spatial Channel Model (SCM) channel (described in Appendix A), which includes the blockage loss $B L(t)$ (described in Appendix B), $z_{k}(t) \sim$ $\mathcal{C N}\left(0, \sigma_{z}^{2}\right)$ is the noise seen at the $k$-th receiver and $\mathbf{b}_{T x}$ and $\mathbf{b}_{R x}$ are the Tx and Rx beamforming vectors used in forming the beam pair.

In the NR standard, the initial beam pair is established during the Initial Access (IA) phase with the beam management Procedure 1 (P1), consisting of dual Tx and Rx beams sweeping [28]. During this procedure, the BSs periodically broadcast synchronisation signal blocks (SSBs), sweeping all the Tx beams over successive SSBs and repeating the same operation $N_{\mathrm{CB}, R x}$ times. On the other hand, the UE sweeps the Rx beam every $N_{\mathrm{CB}, T x} \mathrm{SSB}$, while measuring the Reference Signal Received Power (RSRP) for each Tx-Rx beam pair. After the BS-UE sweep all the combinations of Tx and Rx beams, the best beam pair, called primary beam pair, is selected based on the maximum RSRP. Thus the UE associates to the BS providing the maximum RSRP computed with beamforming at both $\mathrm{Tx}$ and $\mathrm{Rx}$ antennas [29]. The time to sweep all the Tx-Rx beam pairs and complete an entire cycle of SSBs transmission can be expressed as ${ }^{1}$

$$
T_{\text {sweep }}=T_{S S} \frac{N_{\mathrm{CB}, T x} N_{\mathrm{CB}, R x}}{L_{\mathrm{SSB}}}+\frac{T_{S S}}{2},
$$

where $T_{S S} / 2$ is the average time to wait until the subsequent SS Burst Set transmission, assuming that the blockage happens at a time $\bar{t} \sim \mathcal{U}\left[t, t+T_{S S}\right]$ within the duration of two NR frames.

After the beam-sweeping procedure is completed, the DL transmission between BS $j$ and UE $k$ through the primary beam pair provides a data rate that can be expressed as

$$
r_{j, k}^{\prime}(t)=\frac{B W}{K_{j}} \log _{2}\left(1+\frac{P_{b}\left|\mathbf{b}_{R x}^{\top} \mathbf{H}_{j, k}(t) \mathbf{b}_{T x}\right|^{2}}{I_{j, k}(t)+\sigma_{z}^{2}}\right),
$$

where $K_{j}$ represents the number of UEs served by the BS $j$ and $I_{j, k}(t)$ represents the inter-cell interference, which can be expressed as $I_{j, k}(t)=\sum_{j^{\prime} \in \mathcal{J} \backslash j} \sqrt{P_{b}} s_{k^{\prime}}(t) \mathbf{b}_{R x}^{\top} \mathbf{H}_{j, k}(t) \hat{\mathbf{b}}_{T x}$, where $\hat{\mathbf{b}}_{T x}$ represents the Tx beam directed from BS $j^{\prime}$ to UE $k^{\prime} \neq k$.

\section{BEAM RECOVERY METHODS}

In what follows, we consider that the primary beam pair becomes blocked when the 3D rectangular screen modelling the blocker intersects the LoS path between BS $j$ and UE $k 2^{2}$ At time $t$, the Ground Truth (GT) state of the Tx beam $l$, forming the primary beam pair, can be expressed as

$$
S_{l}(t)=\left\{\begin{array}{lll}
0 & \text { (non-blocked) } & \text { for } 0<t<\bar{t} \cup t \geq \bar{t}+T_{b l}, \\
1 & \text { (blocked) } & \text { for } \bar{t} \leq t<\bar{t}+T_{b l},
\end{array}\right.
$$

where $T_{b l}$ represents the blockage event duration.

In the NR standard, the status of the beam is monitored through the RSRP measurements, based on the Channel State Information - Reference Signals (CSI-RSs) received at the UE, and reported periodically back to the BS using the Uplink (UL) control channels [24], [30]. The RSRP values can be mapped to the SNR through a linear relationship ${ }^{3}$ For instance, $\operatorname{SNR}[\mathrm{dB}]=\operatorname{RSRP}[\mathrm{dBm}]+122 \mathrm{dBm}$, when the receiver noise is $-122 \mathrm{dBm}$. Thus, we consider to track the quality of the primary beam pair with the instantaneous SNR, expressed as $\operatorname{SNR}_{l}(t)=\frac{P_{b}\left|\mathbf{b}_{R x}^{\dagger} \mathbf{H}(t) \mathbf{b}_{T x}\right|^{2}}{\sigma_{z}^{2}}$ and the average value of SNR for the non-blocked time instants denoted as $\overline{\mathrm{SNR}}_{l}$.

When the primary link becomes blocked, a neighbour BS in proximity of the coverage area of the server BS, can provide

${ }^{1}$ Each SSBs is mapped to 4 OFDM symbols of the TTI in the time domain and $20 \mathrm{RBs}$ over $275 \mathrm{RBs}$ in the frequency domain [24]. Multiple SSBs are grouped in a SS Burst and cover successive TTIs. Multiple SS Bursts are referred to as SS Burst Set, which is transmitted in the first half-frame (5 $\mathrm{ms})$ and has a periodicity of two NR frames $\left(T_{S S}=20 \mathrm{~ms}\right)$. The maximum number of SSBs within each SS Burst Set is frequency-dependent and is equal to $L_{\mathrm{SSB}}=64$ for the Frequency Range 2 (FR2) [30].

${ }^{2}$ This assumption is primarily motivated by the indoor scenario, where we measured a probability of having a LoS link between the BS server and UE that is $99.9 \%$

${ }^{3}$ The mapping between RSRP and SNR is valid under the assumption that the RSRP does not account for the inter-cell interference, since it is computed with the average power of all the cell-specific CSI-RSs carried over multiple RBs. 


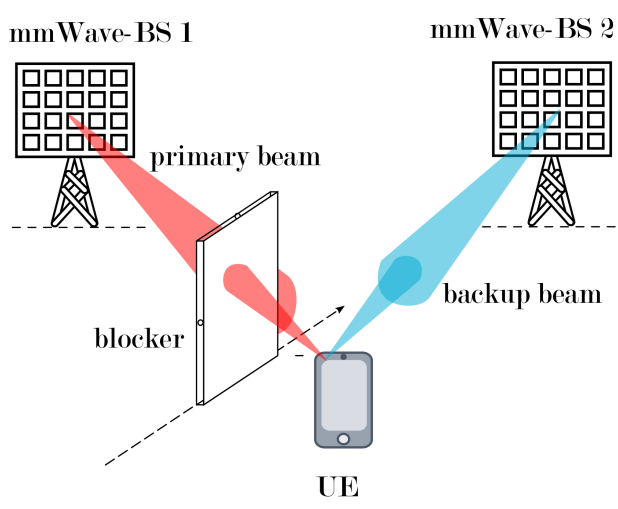

(a) Dynamic blockage scenario with primary and backup beams represented for one of the UE.

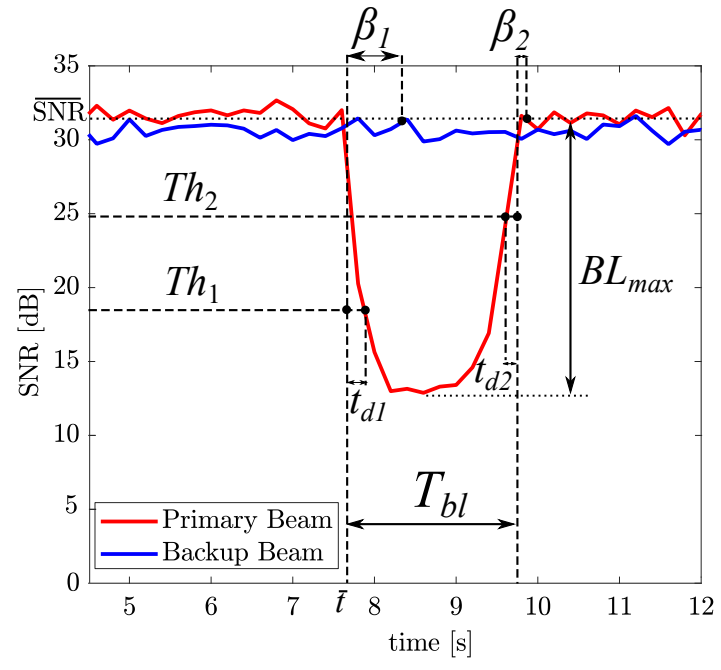

(b) Temporal variations of the SNR for primary and backup beams during the blockage event.

Fig. 3: Beam switching operations of the method based on detection according to the temporal variations of the SNR values for primary and backup beams.

a backup beam pair from a different spatial direction of the primary beam pair and most likely not affected by the blockage when the primary is blocked, as shown in Fig. 3a. Hence, we consider that the UE identifies a secondary BS, for instance, during the IA phase, which provides the second-largest RSRP computed adopting beamforming at both $\mathrm{Tx}$ and $\mathrm{Rx}$ antennas. We assume to repeat the beam sweeping procedure to identify the backup beam pair at every blockage event since, in dynamic environments, the beam training results may not stay the same for a long time [13]. Additionally, we assume that data transmission occurs during the beam sweeping procedure $T_{\text {sweep }}$. This is because, as described before in Sec. II-B, the SSBs use limited spectrum resources, i.e. 20 RBs over specific OFDM symbols, and the remaining spectrum resources may be dedicated to data transmission $[31]^{4}$

\section{A. Beam Recovery Based on Blockage Detection}

In this section, we describe a potential implementation of the current state-of-art method based on a detection threshold to recover the beam when the mmWave links are affected by blockage events, as represented in Fig 3 a . The beam recovery based on the detection (BR-Det) method is considered the benchmark for our studies and operates in three phases. Firstly, it identifies the blockage events when the variations of DL SNR surpass a threshold. Then, it recovers the blocked beams after aligning the backup beam pair with the secondary BS, and finally, it switches back to the primary beam once the blockage is cleared. We define as $T h_{1}$ and $T h_{2}$ two thresholds with values corresponding to $\overline{\mathrm{SNR}}_{l}$ minus $70 \%$ and $30 \%$ of

\footnotetext{
${ }^{4}$ The data transmission in the SSB TTIs is possible under certain circumstances. For instance, the secondary BSs may serve those UEs in the same direction of the SSB transmission [31], whereas the serving BSs may schedule the UE in different TTIs from the ones used by the SSBs or the UE may perform beam sweeping within the TTI used for SSB transmissions.
}

the maximum blockage loss $B L_{\max }$, respectively, in line with the procedure adopted in [23].

As shown in Fig. 3b, whenever the beam is non-blocked, the SNR shows small fluctuations around the average value $\overline{\mathrm{SNR}}_{l}$ due to the channel's multipath components. At the time instant $\bar{t}$ when the blocker starts to intersect the primary beam, the SNR starts to decay rapidly, and the blockage event is detected if $\mathrm{SNR}_{l}(t)<T h_{1}$. The beam sweeping procedure follows this event to align the backup beam pair. The time that it takes to handover the communication to the secondary BS from the time when the blockage begins can be expressed as

$$
\beta_{1}=t_{d 1}+T_{\text {sweep }}+T_{H O},
$$

where $t_{d 1}$ is the interval between $\bar{t}$ and the time instant when the SNR decays below the threshold $T h_{1}, T_{\text {sweep }}$ defined in Eq. (2) denotes the beam sweeping duration to identify the backup beam pair and $T_{H O}$ represents the time interval before the handover to the secondary BS. After switching to the backup beam pair, the data rate of the DL transmission between the secondary BS $j^{\prime}$ and UE $k$ can be expressed as

$$
r_{j^{\prime}, k}^{\prime \prime}(t)=\frac{B W}{K_{j^{\prime}}} \log _{2}\left(1+\frac{P_{b}\left|\overline{\mathbf{b}}_{R x}^{\top} \mathbf{H}_{j^{\prime}, k}(t) \overline{\mathbf{b}}_{T x}\right|^{2}}{I_{j^{\prime}, k}(t)+\sigma_{z}^{2}}\right),
$$

where $\overline{\mathbf{b}}_{T x}$ and $\overline{\mathbf{b}}_{R x}$ denote the Tx and Rx beamforming vectors of the backup beam pair.

At the end of the blockage event, which lasts for the duration $T_{b l}$, the primary beam SNR returns to values without blockage, i.e. $\overline{\mathrm{SNR}}_{l}$, and the UE switches back to the primary beam pair. The time that it takes to handover the communication back to the BS $j$ from the time when the blockage ends can be expressed as

$$
\beta_{2}=T_{H O}-t_{d 2},
$$


where $t_{d 2}$ is the interval between the time instant $\bar{t}+T_{b l}$, representing the end of the blockage and the time instant when $\operatorname{SNR}(t)>T h_{2}$, and $T_{H O}$ is the time before the handover to BS $j$.

The data rate of UE $k$ obtained while adopting the BR-Det method can be expressed as

$$
r_{k}^{\mathrm{BRDet}}(t)= \begin{cases}r_{j, k}^{\prime}(t) & \text { for } 0<t<\bar{t}+\beta_{1}, \\ r_{j^{\prime}, k}^{\prime \prime}(t) & \text { for } \bar{t}+\beta_{1} \leq t<\bar{t}+T_{b l}+\beta_{2}, \\ r_{j, k}^{\prime}(t) & \text { for } t \geq \bar{t}+T_{b l}+\beta_{2} .\end{cases}
$$

\section{B. Beam Recovery Based on Blockage Predictions}

In this section, we propose a novel beam recovery method based on predictions (BR-Pre) capable of switching to the backup beam pair, before the blocker affects the quality of the ongoing communication over the primary beam pair.

Firstly, we define the prediction window $\eta$ to be larger than the beam sweeping duration $T_{\text {sweep }}$ and the handover period $T_{H O}$, i.e. $\eta>T_{\text {sweep }}+T_{H O}$. The BR-Pre method evaluates at the beginning of the prediction window, i.e. at the time $t-\eta$, the beam state predictions $\hat{S}_{l}$ indicating whether the blockage is going to obstruct the primary beam after the end of the prediction window $\eta$, at time $t$. If the beam is predicted as non-blocked, i.e. $\hat{S}_{l}(t)=0$, the BR-Pre method continues using the primary beam pair. Differently, when the beam is predicted as blocked, i.e. $\hat{S}_{l}(t)=1$, the BR-Pre method uses this early indication to start the beam sweeping procedure in advance to establish the backup beam pair 5

At the end of the prediction window, the BR-Pre method switches to a newly computed backup beam pair and the data rate of the DL transmission between the secondary BS $j^{\prime}$ and UE $k$ can be expressed as in Eq. 6. We want to emphasise that the advantage of the BR-Pre against the BR-Det method is that at the time of the beam switching (assuming the beam state predictions are correct, i.e. $\left.\hat{S}_{l}(t)=S_{l}(t)\right)$ the primary beam SNR has not yet dropped since the beam switching happens at the end of the prediction window and before the blockage begins, while with the BR-Det method described in Sec. III-A. the beam switching happens with a delay $\beta_{1}$ after the blockage begins.

During the blockage, the BR-Pre method continues to evaluate the predictions of the beam $l$, and if the state becomes non-blocked, i.e. $\hat{S}_{l}(t)=0$, the BR-Pre switches back to the primary beam pair. The data rate of UE $k$ obtained while adopting the BR-Pre method can be expressed as

$$
r_{k}^{\text {BRPre }}(t)= \begin{cases}r_{j, k}^{\prime}(t) & \text { if } \hat{S}_{l}(t)=0, \\ r_{j^{\prime}, k}^{\prime \prime}(t) & \text { if } \hat{S}_{l}(t)=1 .\end{cases}
$$

\section{BeAm State Predictions}

In this section, we propose a procedure to obtain the beam state predictions $\hat{S}_{l}$ adopted in the BR-Pre method. The main

\footnotetext{
${ }^{5}$ We recall that the beam sweeping procedure can be performed during data transmission as it only uses a partial set of RBs and OFDM symbols in the TTIs where the SSB transmission occurs.
}

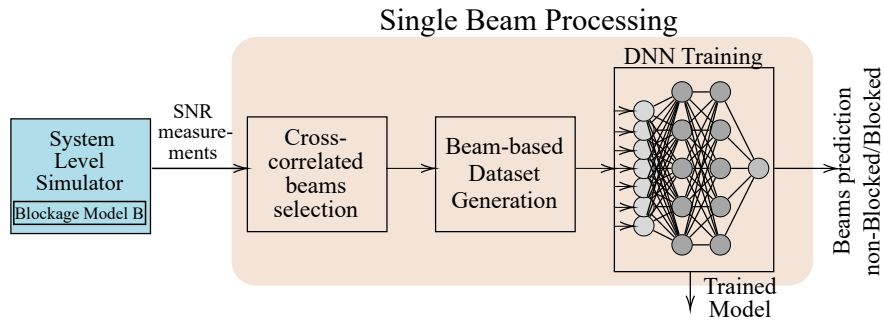

Fig. 4: Key steps of the procedure used to obtain the beam state predictions.

idea is to use the SNR measurements of other Tx-Rx beam pairs - exchanged between BSs through a central server - to predict $\eta$ time instants before the blockage changes the state of the beam $l$ at time $t$. As the blockage effect follows in general non-periodic temporal dynamics, the SNR does not change until the blocker intersects the beam $l$, making it unpractical to infer the beam-state only by analysing the SNR temporal variations of the single beam. On the other hand, by looking at the SNR temporal variations of other beams, their SNR may drop earlier than the SNR of the beam to predict. We train a DNN model to relate these early SNR variations of other beams (inputs) to the blockage states of the beam $l$ in successive instants of time (output).

Furthermore, as the BS is adopting a codebook with multiple beams, the DNN model needs to simultaneously provide multiple beam state predictions. For example, with a codebook formed by two beams, the first label indicates the state of the first beam (blocked, non-blocked) while the second label indicates the state of the second beam, assumed independent of the state of the first beam. Therefore, the task consists of learning one binary classifier per beam, meaning that we train and validate a beam-specific DNN model for each beam. As shown in Fig. 4, a sequence of steps leads to the beam-specific prediction $\hat{S}_{l}$. We describe each step in the sections that follow.

\section{A. Cross-correlated Beams Selection}

In this subsection, we introduce a pre-processing step that extracts a subset of beams to use for the predictions considering the cross-correlation between the SNR time series of different beams. This procedure is motivated by two observations. First, the network presents many beams that will be irrelevant for predicting a specific beam state. This could needlessly complicate the training process without delivering any improvement. Second, since correlated beams are most likely spatially close, they also belong to the same BS or neighbour BSs. Therefore, we can also optimise the exchange of information among BSs to limit the use of a central server.

Let us consider the pair of Tx beams $l-l^{\prime}$. The delay between $l$ and $l^{\prime}$ is estimated as $\delta_{l, l^{\prime}}=\arg \max _{\forall \tau} R_{l, l^{\prime}}(\tau)$, where $R_{l, l^{\prime}}(\tau)=\sum_{t=0}^{T} \operatorname{SNR}_{l}(t) \operatorname{SNR}_{l^{\prime}}(t-\tau)$ is the cross-correlation between the SNR time series of $l$ and the SNR time series of $l^{\prime}$ defined over the time window $[0, T]$, where $T$ is the total observation time. We estimate the delays between $l$ and all the other beams $l^{\prime} \in \mathcal{B} \backslash l$, where $\mathcal{B}=\left\{1,2, \ldots,\left(N_{\mathrm{CB}, T x} \times J\right)\right\}$ 


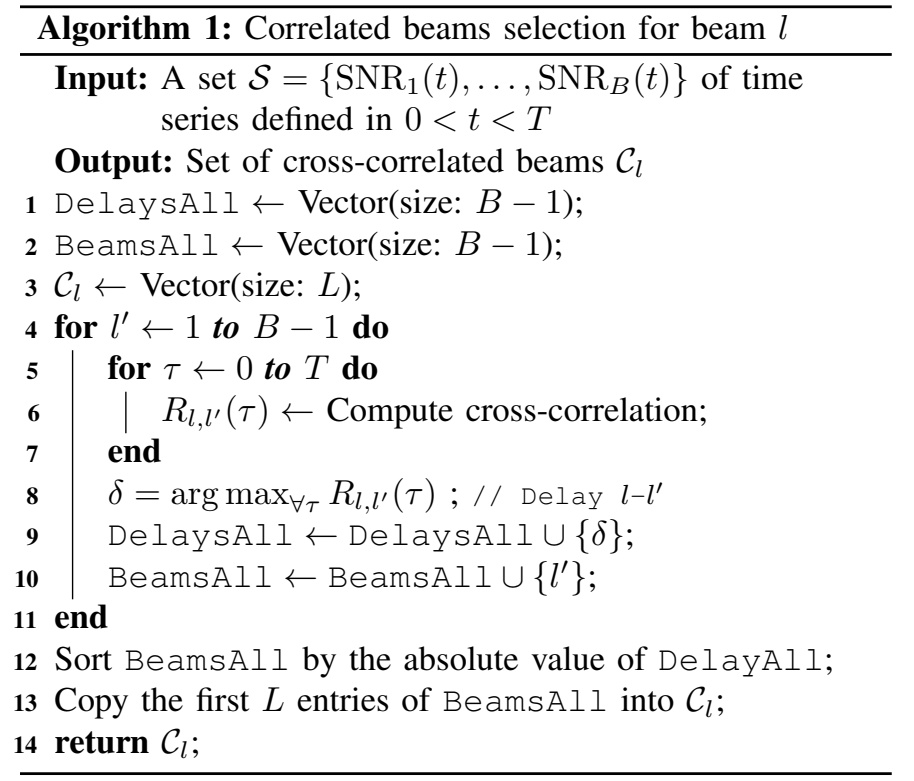

represents the set of all the network's beams and $B=$ $N_{\mathrm{CB}, T x} \times J$ is the total number of beams in the network. Then, we pick the $L$ beams that have the lowest delays in absolute value, indicating that they are spatially close to beam $l$ and we define a set $\mathcal{C}_{l}=\left\{c_{1}, c_{2}, \ldots, c_{L}\right\}$, which contains the beams to use for predicting the state of beam $l$. In Algorithm 1. we describe the procedure in details.

\section{B. Dataset Generation}

We consider a dataset formed by $N$ samples, where the $n$ th sample of the dataset is organised into input/output vectors generated as follows:

- Input: let us define as $\mathbf{v}_{l}=\left[\mathrm{SNR}_{l}[t-\eta-(\epsilon-\right.$ 1)], $\left.\mathrm{SNR}_{l}[t-\eta-(\epsilon-2)], \ldots, \mathrm{SNR}_{l}[t-\eta]\right]^{\top}$ the vector containing $\epsilon$ samples of the SNR for beam $l$. We assume that the SNR measurements from the beams in the set $C_{l}=\left\{c_{1}, c_{2}, \ldots, c_{L}\right\}$ are exchanged with the BSs through a central server. These data are also represented for each beam $l^{\prime} \in \mathcal{C}_{l}$ with a vector $\mathbf{v}_{l^{\prime}}=\operatorname{SSNR}_{l^{\prime}}[t-$ $\left.\eta-(\epsilon-1)], \mathrm{SNR}_{l^{\prime}}[t-\eta-(\epsilon-2)], \ldots, \mathrm{SNR}_{l^{\prime}}[t-\eta]\right]^{\top}$ containing $\epsilon$ samples of the SNR. Thus, the input is defined as $\mathbf{X}_{n, l}=\left[\mathbf{v}_{l}, \mathbf{v}_{c_{1}}, \mathbf{v}_{c_{2}}, \ldots, \mathbf{v}_{c_{L}}\right] \in \mathbb{R}^{\epsilon \times(L+1)}$ and aggregates the SNR measurements of $L+1$ beams altogether.

- Output: the output is constituted by a variable $Y_{n, l}$ set to 1 (blocked) or 0 (non-blocked) according to the GT state of the beam $l$ at time instant $t$. We obtain this information from the simulation environment. We set the label to 1 (blocked), when the blocker intersects the LoS path between BS and UE. Conversely, we set the label to 0 (non-blocked) when there is no intersection.

If the beam is assigned to multiple UEs, we fill in the input vector with the median SNR and output variable corresponding to the most frequent label inside the UEs group. If there are no UEs assigned to a beam, the corresponding SNR
TABLE I: DNN network parameters

\begin{tabular}{|c|c|c|}
\hline \multirow{4}{*}{$\begin{array}{c}\text { Network } \\
\text { architecture }\end{array}$} & Number of layers & 2 (Fully-connected) \\
\cline { 2 - 3 } & Number of neurons & 20 \\
\cline { 2 - 3 } & Weights initialisation & He \\
\cline { 2 - 3 } & Activation function & ReLu \\
\cline { 2 - 3 } & Dropout & None \\
\cline { 2 - 3 } Training \\
options & Normalisation Input layer & "Zero-center" \\
\cline { 2 - 3 } & Solver name & Adam \\
\cline { 2 - 3 } & Mini-batch size & 1000 \\
\cline { 2 - 3 } & Number of Epochs & 50 \\
\cline { 2 - 3 } & Learning rate & $10^{-3}$ \\
\hline \multirow{4}{*}{} & L2 regularisation & $10^{-5}$ \\
\hline
\end{tabular}

measurement cannot be recorded, and the input's entries are filled with a constant SNR (in our simulations, we use $60 \mathrm{~dB}$ ) outside the range of the possible SNR values.

Figs. $5 \mathrm{a}$ and $5 \mathrm{~b}$ show a simplified scenario where a set of beams shown on the left side of the BS is used to predict the state of the beam $l$, depicted with the line pattern (magenta) on the right side of the BS. The entries of the dataset are formed by associating the inputs $\mathbf{X}_{1, l}$ and $\mathbf{X}_{2, l}$ to the values of the GT state $Y_{1, l}$ and $Y_{2, l}$ reported in Fig. 5c. The two different examples show how the input data changes depending on the value of the ground-truth. In the first example, depicted in Fig. 5a the SNR measurements (within the sliding window of length $\epsilon$ ) show small variations indicating that the set of beams at the time $t_{1}-\eta$ is not affected by the blocker. This behaviour suggests that also the magenta beam is likely not affected by the blocker at the instant $t_{1}$. Indeed, as shown in Fig. 5c the GT state corresponds to the non-blocked value, i.e. $Y_{1, l}=0$. On the other hand, in the second example, depicted in Fig. 5b, the SNR measurements show large drops for the set of beams at the time $t_{2}-\eta$, indicating that the magenta beam is likely affected by the blocker movement at the time $t_{2}$. This is confirmed by the GT state $Y_{2, l}=1$, which corresponds to the blocked value.

\section{Deep Neural Network Model Training}

For training the ML model, we employ a DNN structure constituted by: $i$ ) an input layer with $\epsilon \times(L+1)$ inputs, $i i)$ two hidden layers, and iii) a softmax function as output layer. We train each DNN model with ADAptive Moment estimation (Adam) optimiser adopting He weights initialisation and Rectified Linear unit (ReLu) activation functions [32], [33].

During the training, we divide the dataset into mini-batch of size $N_{b s}$ assuming a constant learning rate. We repeat the training for several epochs to find the set of model parameters denoted as $\boldsymbol{\Omega}_{l}$ that minimises the cross-entropy loss, given the set of inputs $\mathbf{X}_{1, l}, \ldots, \mathbf{X}_{N_{b s}, l}$ and the set of GT labels $Y_{1, l}, \ldots, Y_{N_{b s}, l}$. The lower is the loss of the model output compared to the ground-truth, and the better is the model.

Moreover, since the time spent by the beam in the nonblocked state prevails over the time spent in the blocked state, the dataset presents a skewed distribution towards the nonblocked class that implies a class imbalance problem [35]. To re-balance the loss, we assign a higher weight to the crossentropy loss computed for the rare class (blocked) and a lower weight to the cross-entropy loss computed for the dominant 

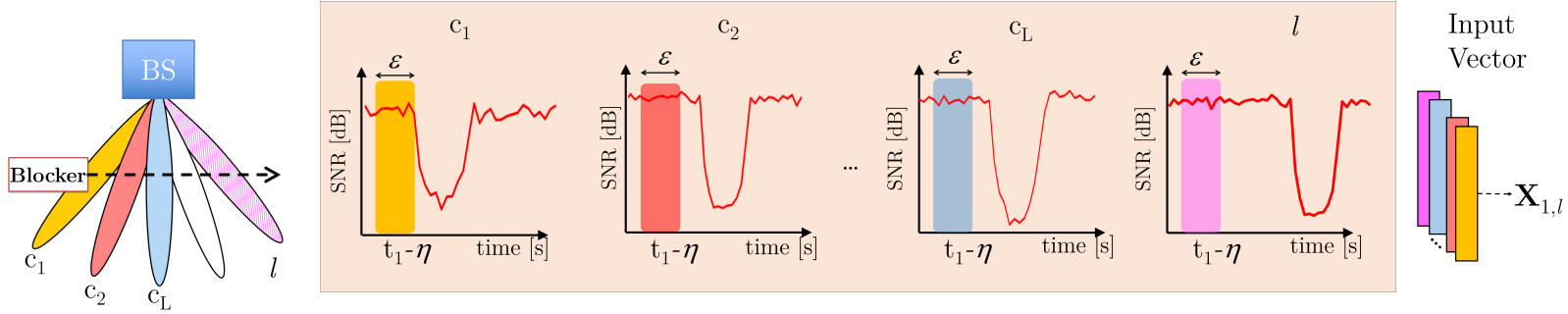

(a) Example of input data corresponding to the non-blocked output state.
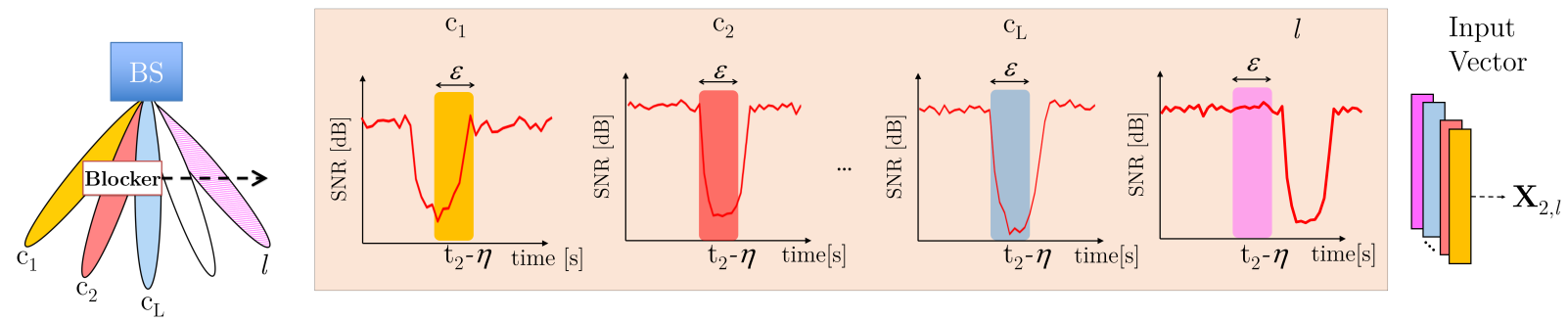

(b) Example of input data corresponding to the blocked output state.

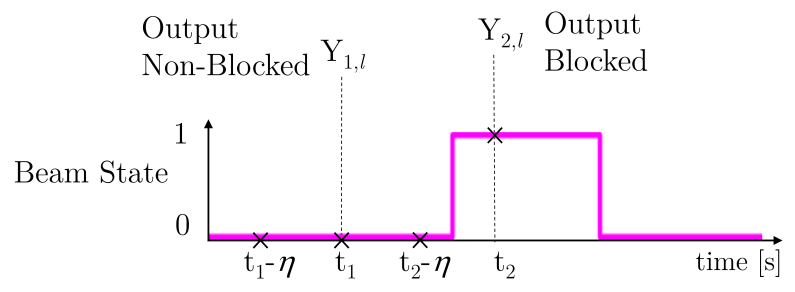

(c) Examples of output data for non-blocked and blocked states of the magenta beam.

Fig. 5: Two examples of input-output vectors forming the dataset for the beam depicted with the line pattern (magenta) on the left side of Figs 5a and 5b In Fig. 5a the input data are taken at a time $t_{1}-\eta$ from the SNR measurements and correspond to the non-blocked state at the time $t_{1}$ in Fig. 5c . Conversely, in Fig. 5b the input data are taken at a time $t_{2}-\eta$ and correspond to the blocked state at the time $t_{2}$ in Fig. $5 \mathrm{c}$

class (non-blocked). The weighted formulation of the crossentropy loss can be expressed as

$$
\begin{aligned}
\mathcal{L}_{l}(\boldsymbol{\Omega})=-\frac{1}{N_{b s}} \sum_{n=1}^{N_{b s}} \mu_{1, l} Y_{n, l} \log \left(\widehat{Z}_{n, l}(\boldsymbol{\Omega})\right) & \\
& +\mu_{2, l}\left(1-Y_{n, l}\right) \log \left(1-\widehat{Z}_{n, l}(\boldsymbol{\Omega})\right),
\end{aligned}
$$

where $\widehat{Z}_{n, l}$ is the output of the softmax function and indicates the likelihood for the beam $l$ to be in the state non-blocked or blocked. Moreover, the class weights $\mu_{1, l}$ and $\mu_{2, l}$ are computed as the inverse of the number of training samples for each class, i.e. $\mu_{1, l}=N / \sum_{n=1}^{N}\left[Y_{n, l}=0\right]$ and $\mu_{2, l}=N / \sum_{n=1}^{N}\left[Y_{n, l}=1\right]$, respectively.

Table I] reports the training configurations and the list of hyper-parameters such as the number of hidden neurons, learning rate and L2 regularisation with the initial configuration values we adopted for the training. Note that the L2 regularisation incorporates in the loss function depicted in Eq. (10) a penalty term on the neuron weights, which reduces the overfit to the training dataset and improves the generalisation of the DNN model [36].

\section{EVALUATION}

In this section, we first describe the setup used for generating the synthetic data and preparing the dataset for the training. Then, we validate the results of the DNN model state predictions for multiple beams using a training dataset. Finally, we deploy the DNN models online into the simulator to study the performance of our proposed BR-Pre method and compare with the performance of the BR-Det method, which we consider as our benchmark.

\section{A. Experiment Setup}

The preparation of the dataset used for training the DNN models involves several steps that entail the setup of the 3GPP-based system-level simulator, including the setup of the blocker's movement, the generation of a beam-specific dataset, the selection of the validation metric and the configuration of the prediction parameters. Now we discuss each of these steps: 


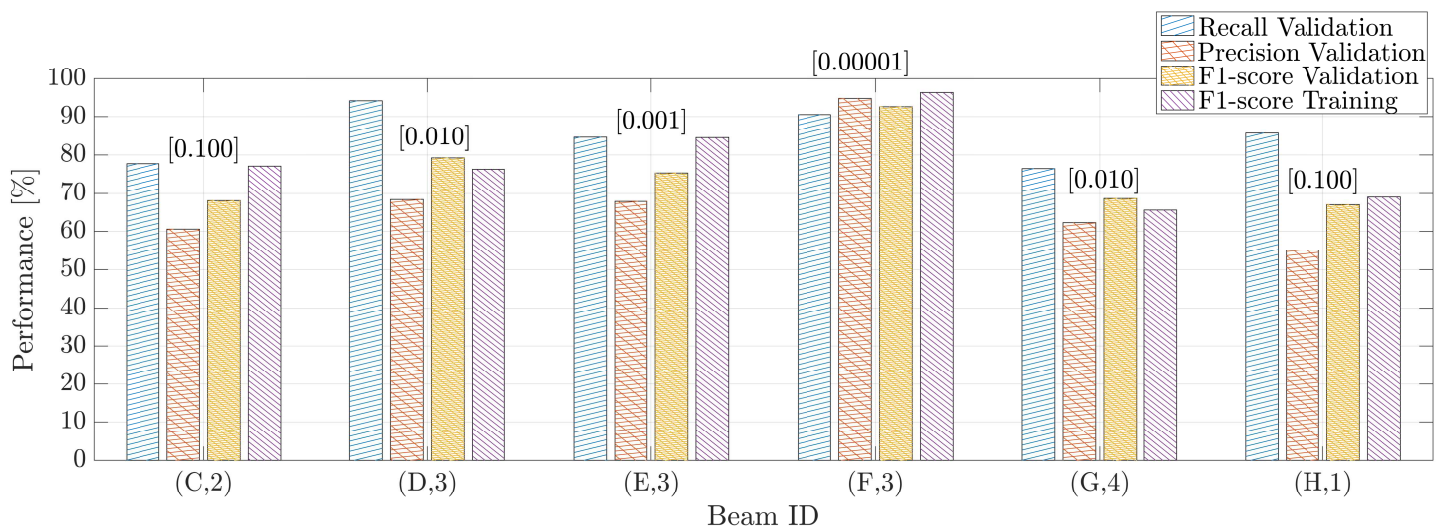

Fig. 6: Training results of the DNN models for blockage state prediction for several beams of BS-3.

1) Simulation setup: we take a drop-based approach to generate synthetic data utilising a detailed and calibrated system-level simulator that implements 3GPP 3D SCM for the $0.5-100 \mathrm{GHz}$ spectrum [25]. We configure the simulator according to the list of parameters reported in Table II of Appendix C. We simulate the mmWave network shown in Fig. 1. placing the BSs on a rectangular grid and the UEs in random positions, which are kept fixed between drops. We assume time-invariant large-scale channel parameters (LSPs) such as delay spread, AoD and AoA spread, Rician K-factor, shadow fading (SF) and pathloss, for the duration of the drop $T$. Additionally, at each simulation step within a drop, we assume $i)$ time-variant blockage loss $B L(t)$ due to the blocker's movement and ii) time-variant small-scale channel parameters (SSPs), e.g. power, phase, delay, AoA and AoD for each MPC, that change independently between simulation steps due to the variations of the multipath fading. We fix the network drop duration to $T=40 \mathrm{~s}$, and we consider an interval $\Delta t=200 \mathrm{~ms}$ to update the channel $\mathbf{H}$ between the simulation steps. We compute every BS-UE link's channel and the DL SNR for each beam and for all the RBs of the system bandwidth at each simulation step.

2) Dynamic blockage generation: to model the blockage loss, we adopt the Blockage Model B that is incorporated within the 3GPP 3D SCM. Compared to the stochastic Blockage Model A [37], the deterministic Blockage Model B supports spatial consistency, which enables to simulate the smooth and continuous-time evolution of the blockage effect while capturing the correlated behaviour of the blockage loss for UEs located closely [25]. As a case study, we consider the presence of one blocker at a time moving in the network scenario. The blocker has dimensions $2 \mathrm{~m} \times 3 \mathrm{~m}$ and constant speed $v$ selected randomly between two values $1 \mathrm{~m} / \mathrm{s}$ and 2 $\mathrm{m} / \mathrm{s}$, in line with the recommended blocker parameters listed in [25]. The initial position is set at the $\mathrm{x}-\mathrm{y}$ coordinates $(-20,0)$ $\mathrm{m}$, corresponding to the left side of the network layout, and it moves along the $\mathrm{x}$-axis line from left to right till reaching $\mathrm{x}-\mathrm{y}$ coordinates $(20,0)$. When the blocker arrives at the right side of the network layout, it regenerates at the initial position and repeats the same movement ${ }^{6}$

3) Beam-specific dataset: we generate a training dataset per beam, collecting SNR measurements over time for successive simulation steps and repeat the same process for 100 network drops, including both values of the blocker speed in the same dataset. For instance, the beam $(\mathrm{C}, 2)$ dataset has $\sim 1.5$ million samples divided between $\sim 80$ thousand samples for the blocked class and $\sim 1.42$ million samples for the nonblocked class. We split this data according to the commonly used ratio of $80 / 20$ to use $80 \%$ of the dataset for training the DNN model and the remaining $20 \%$ of the dataset to validate the DNN model performance. During the training, we consider mini-batch of size $N_{b s}=1000$ samples and 50 training epochs.

4) Validation metrics: as shown before for the beam $(\mathrm{C}, 2)$, the number of non-blocked samples significantly outweighs the blocked samples. Thus, for our specific scenario, we validate the DNN models adopting the F1-score metric, which is more appropriate than the Accuracy metric for the problems presenting highly unbalanced class-distribution [35]. The F1score combines through the harmonic mean Precision and Recall and can be calculated as F1-score $=\frac{2 \times \text { Precision } \times \text { Recall }}{\text { Precision }+ \text { Recall }}$. Recall indicates the proportion of actual blocked samples (True Positive (TP) cases) over all the blocked samples (TP and False Negative (FN) cases), and is calculated as Recall $=\frac{\mathrm{TP}}{\mathrm{TP}+\mathrm{FN}}$. Conversely, Precision indicates the proportion of actual blocked samples among all predicted blocked samples (TP and False Positive (FP) cases) and is calculated as Precision $=\frac{\mathrm{TP}}{\mathrm{TP}+\mathrm{FP}}$.

5) Prediction parameters: we fix the prediction window length to $\eta=400 \mathrm{~ms}$, which is larger than the combined duration of the beam sweeping procedure, i.e. $T_{\text {sweep }}=330$ $\mathrm{ms}$, and the handover time, i.e. $T_{H O}=50 \mathrm{~ms}$ [38]. We adjusted the DNN models input size after several training attempts, which led us to use the number of input beams $L=5$ and set the sliding window duration to $\epsilon=2 \mathrm{~s}$.

\footnotetext{
${ }^{6}$ This use case is indicated for scenarios such as future industrial factories, where Automated Guided Vehicles (AGVs) or industrial robots, may maintain similar movement patterns and follow a dedicated pathway in the factory corridor.
} 


\section{B. Multi-beam Prediction Results}

In Fig. 6, we show the training and validation results of the DNN models taking as an exemplary reference BS-3 and several of its blocked beams having different azimuth and elevation AoDs. Nevertheless, the same process can be repeated without loss of generality for other BSs of the network. The training process highlighted that the DNN models of different beams should be tuned separately. In other words, it is not possible to use the same set of hyper-parameters for all DNNs. Hence, we perform an independent validation of the DNN models beam by beam. During the training, we change the L2 regularisation value in the range $\left\{10^{-5}-10^{-1}\right\}$ to reduce the overfitting. We measure the performance of each beam for five different random initialisation of the neuron weights. Then, we save the model that returns the maximum F1-score performance on the validation data.

Looking at the results in Fig. 6, we report between parenthesis the value of $\mathrm{L} 2$ regularisation hyper-parameter that corresponds to the model attaining the best F1-score. Then, we report Recall, Prediction and F1-score results measured on the validation data and the F1-score result measured on the training data. Firstly, most of the beams show Recall validation performance higher than Precision validation performance. This is due to the class weights applied to the loss function, shown in Eq. [10], which penalises more the FN errors than the FP errors. This setting increases the probability of predicting the blockage correctly but is likely to make more FP errors for the non-blocked samples. Thus the models achieve high Recall while sacrificing their Precision. Secondly, Fig. 6 shows that the beam achieves F1-score validation results close to the F1-score training results. This indicates the models' ability to generalise from the training data to other data generated with the simulator assuming different network drops, the same blocker size/trajectory and blocker speed that can vary randomly between the values of $1 \mathrm{~m} / \mathrm{s}$ and $2 \mathrm{~m} / \mathrm{s}$, as we will see in Sec. $\mathrm{V}-\mathrm{C}$ and Sec. V-E, where we deploy the DNN models into the simulator.

\section{Evolution of the SNR Time Series}

In this section and in the one that follows, we test the DNN models online into the system-level simulator by generating ten different network drops as compared to the ones used in the training dataset with the blocker moving at the speeds $1 \mathrm{~m} / \mathrm{s}$, and other ten network drops with the speed of 2 $\mathrm{m} / \mathrm{s}$. Fig. 7 shows the SNR measurements' evolution in the time domain for two Tx beams of the BS-3's codebook with the blocker moving at the speeds $1 \mathrm{~m} / \mathrm{s}$ and $2 \mathrm{~m} / \mathrm{s}$. These results highlight the BR-Pre behaviour over time and show the differences between the BR-Pre method, the BR-Det method and the case where the transmission continues to be handled by the primary beam. Focusing on Fig. 7a, the bottom part shows the GT and predicted primary beam states. It is worth recalling that the GT state is determined by considering the intersection between the direct path that joins Tx and Rx and the rectangular screen modelling the blocker, as described in

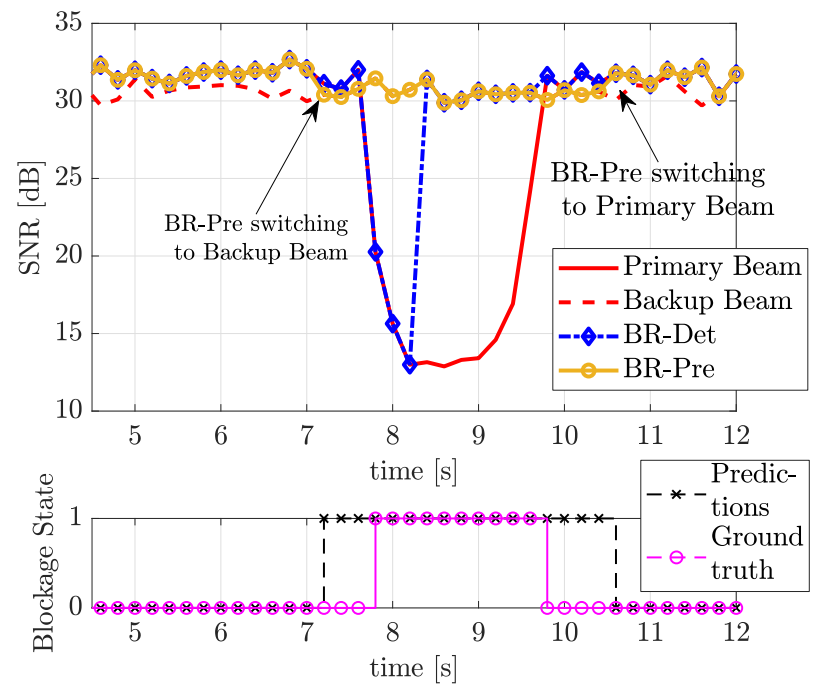

(a) SNR and blockage state for blocker speed $1 \mathrm{~m} / \mathrm{s}$.

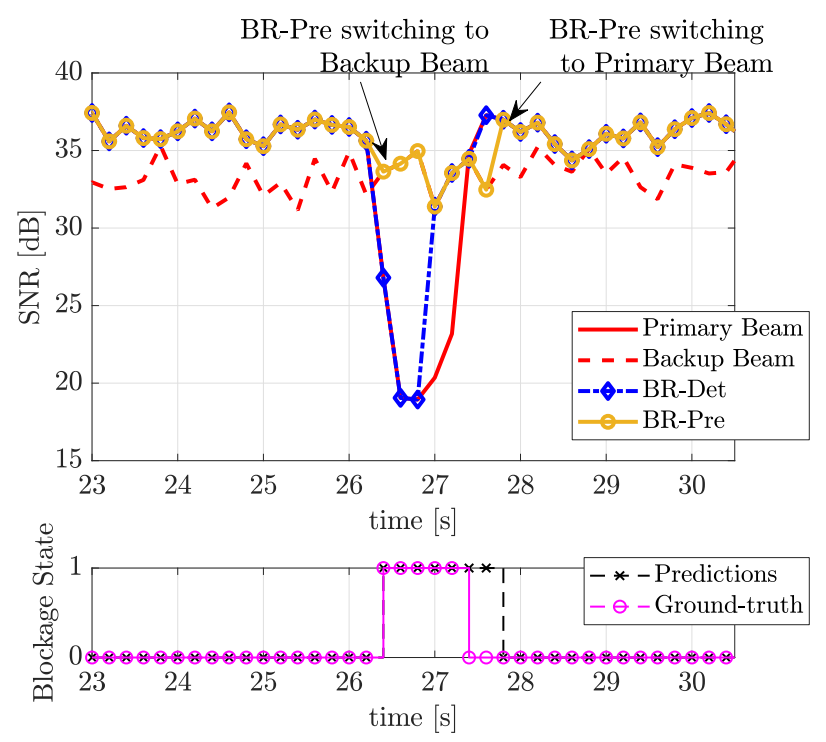

(b) SNR and blockage state for blocker speed $2 \mathrm{~m} / \mathrm{s}$.

Fig. 7: Comparison between the temporal variations of the SNR for the beam recovery methods (top) and blockage state predictions (bottom) for two different beams and blocker speeds.

Section IV-B Conversely, the upper part of Fig $7 \mathrm{a}$ shows the corresponding evolution of the measured SNR.

During the non-blocked time instants, the backup beam pair shows a lower SNR than the primary beam pair because it is established with a secondary BS. Differently, before the primary beam enters into a blocked state (as indicated by the ground-truth state in the bottom part of the figure), the SNR of the primary beam pair starts decreasing, while the SNR of the backup beam pair remains stable, providing a higher SNR than the primary beam for the following blocked time instants. The BR-Det method switches to the backup beam with a delay $\beta_{1}$ that considers the beam sweeping interval initiated after detecting the blockage. Thus, as shown in Fig. 7a, during the 


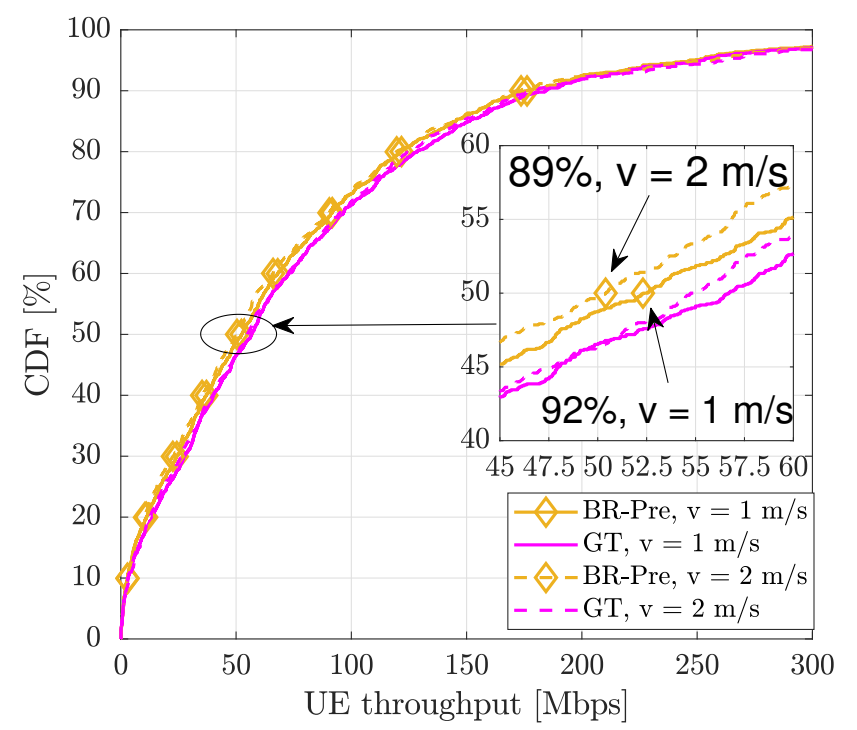

(a) CDF of UEs data rate for blocked time instants.

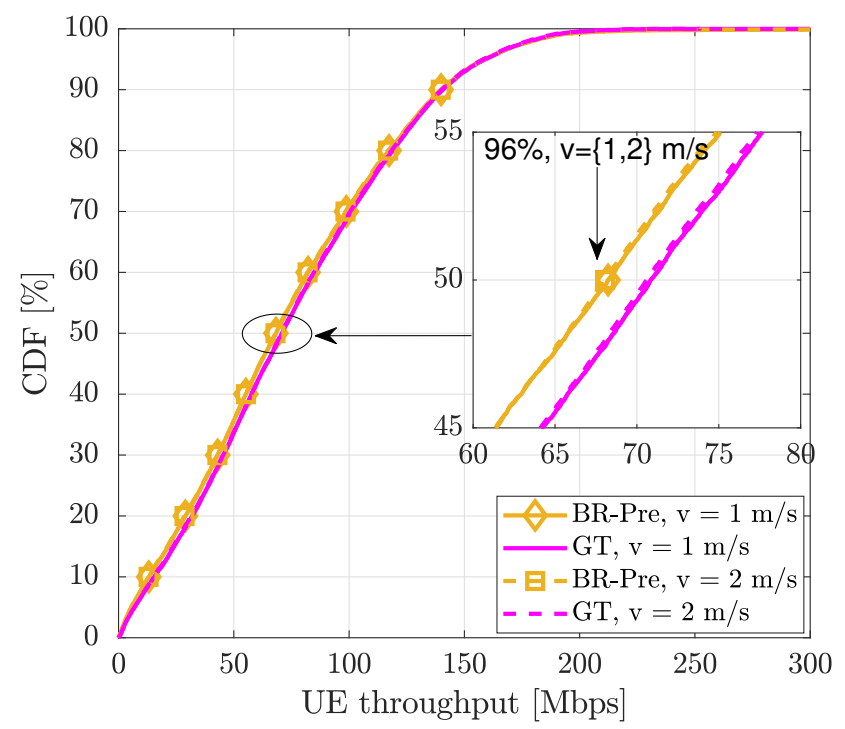

(b) CDF of UEs data rate for non-blocked time instants.

Fig. 8: Data rate performance of the BR-Pre method with online predictions against GT method.

time interval $7.8 \mathrm{~s}-8.4 \mathrm{~s}$, the BR-Det experiences a significant drop of the SNR that is recovered only after switching to the backup beam at the time $t=8.4 \mathrm{~s}$.

On the other hand, as shown in the two Figs. $7 \mathrm{a}$ and $7 \mathrm{~b}$, the BR-Pre method uses the DNN models predictions that infer the beam state $\eta$ time instants ahead of the time instant $t$. Thus, the BR-Pre method initiates the beam sweeping procedure at the beginning of the prediction window, i.e. at the time $t-\eta$. After the end of the prediction window, i.e. at the time $t$, when the SNR of the primary beam pair starts decreasing, the BR-Pre method has already switched to the backup beam and avoids the SNR drop that happens with the BR-Det method. However, as shown in Fig. 7a during the time intervals $7.2 \mathrm{~s}-7.6 \mathrm{~s}$ and $9.8 \mathrm{~s}-10.4 \mathrm{~s}$ and in Fig. $7 \mathrm{~b}$ for the interval $27.4 \mathrm{~s}-27.6$ $\mathrm{s}$ the BR-Pre method presents a SNR lower than the BR-Det method as the BR-Pre method uses the backup beam instead of the primary beam. This is caused by the FP errors associated with the cases when the DNN models wrongly predict the beam state as blocked when there is no blockage present. We will show later in Sec. $\mathrm{V}-\mathrm{D}$ the limited impact of these errors on the data rate performance.

\section{Validation of the BR-Pre Method Performance with Online Predictions}

In this section, we show the cumulative distribution functions (CDFs) of the UE data rate offered by our exemplary BS-3 with the blocker moving at speeds $1 \mathrm{~m} / \mathrm{s}$ and $2 \mathrm{~m} / \mathrm{s}$. In Fig. 8, we show the performance of the BR-Pre method, which uses the DNN models to make online predictions, as explained in Sec. III-B and we compare the results to the GT method, representing the upper bound rate achievable when Eq. (9) has perfect knowledge of the future beam states. During the blocked intervals, the CDFs of Fig. $8 \mathrm{a}$ show that for the blocker speed of $1 \mathrm{~m} / \mathrm{s}$, the median BR-Pre data rate performance reaches $92 \%$ of the GT method performance, while for the blocker moving at $2 \mathrm{~m} / \mathrm{s}$, the BR-Pre method achieves $89 \%$ of the GT data rate. The differences between BR-Pre and GT method data rates are due to the FN errors that cause the BR-Pre method to use the primary beam during the blocked time instants. Nevertheless, these results show that the BR-Pre method almost matches the GT method data rate performance.

On the other hand, Fig. $8 \mathrm{~b}$ shows the CDF of the BR-Pre method data rate achieves $96 \%$ of the median GT data rate during non-blocked time instants due to the impact of the FP errors. The FP errors cause switching to the backup beam during the non-blocked intervals while the primary beam has a larger SNR than the backup beam. Fig. $8 \mathrm{~b}$ show that the different CDFs curves overlap, meaning that the FP errors observed in Fig. 7 do not significantly impact the data rate performance of the BR-Pre method. Overall, the results in Figs. $8 \mathrm{a}$ and $8 \mathrm{~b}$ indicate that the DNN models, when deployed online, generalise well for both the blocker speeds.

\section{E. Data Rate Performance Comparison Between BR-Pre, BR- Det and BF Methods}

In this section, we compare the results between the proposed BR-Pre method, the benchmark BR-Det method reported in Sec. [III-A and the beam fixed (BF) method, presenting the lower bound rate achievable using the primary beam pair. The CDFs in Fig. 9a show that the BR-Pre method outperforms the BR-Det and BF methods during the blocked time instants, especially for the higher blocker speed. These results are explained as follows. We recall that the delay $\beta_{1}$ for detecting and switching the beam penalises the BR-Det data rate at the start of the blockage event. On the other hand, as shown 


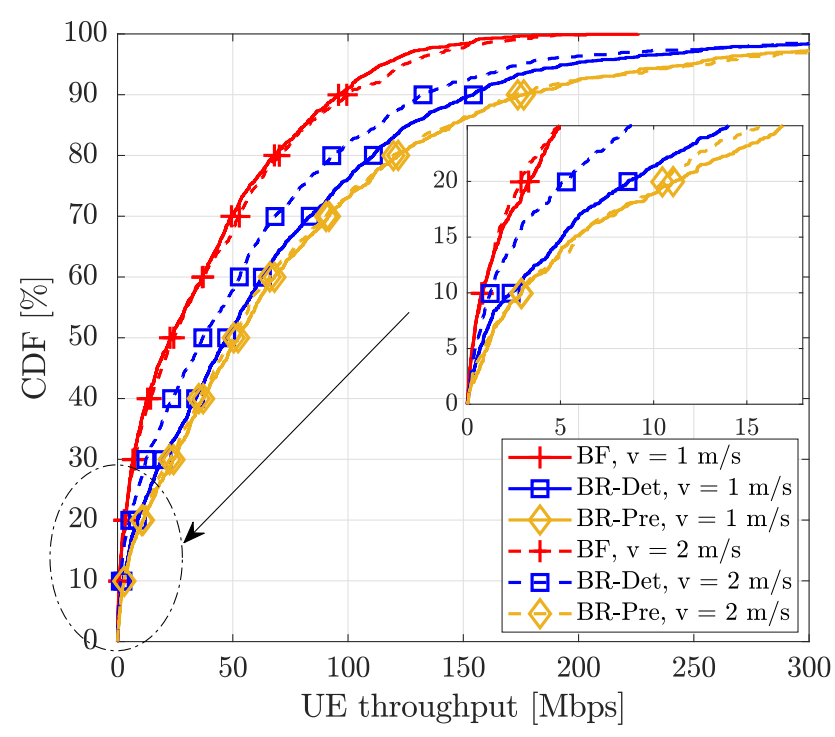

(a) CDF of UEs data rate for blocked time instants.

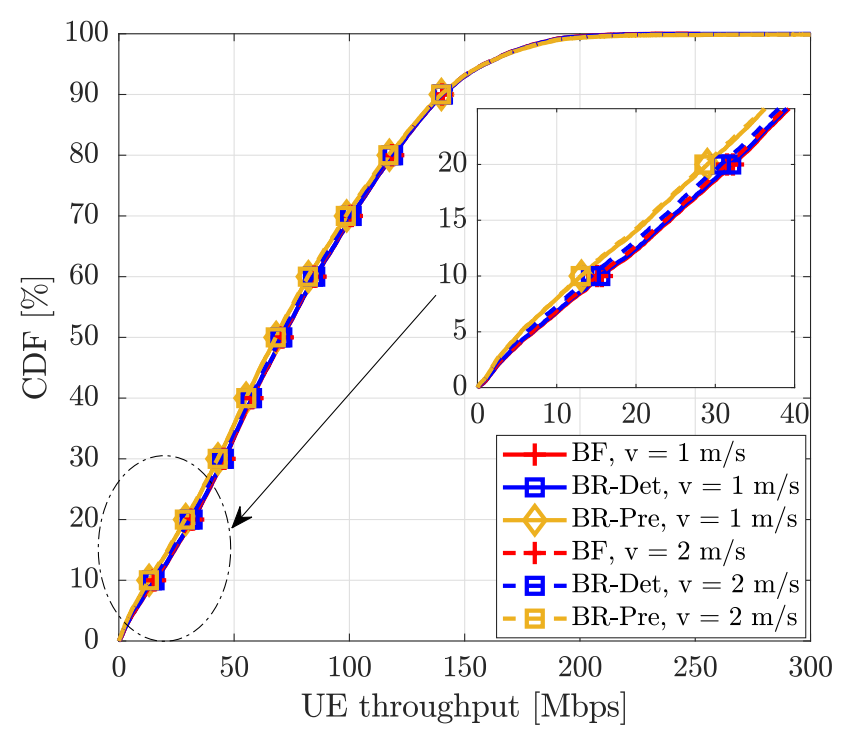

(b) CDF of UEs data rate for non-blocked time instants.

Fig. 9: Data rate performance comparison between BR-Pre, BR-Det and BF methods.

before in Sec. V-D the BR-Pre method performance reaches $92 \%$ and $89 \%$ of the GT data rate due to the low number of FN errors. Overall, the DNN models wrong predictions have a minor impact on the data rate performance than the delay introduced for detecting the blockage and switching the beam. At a higher blocker speed, the beam switching happens more often, and the BR-Det data rate is penalised by the delay more times than with the blocker moving at 1 $\mathrm{m} / \mathrm{s}$, leading to a more significant difference to the BR-Pre results, as represented in Fig. 9a. Additionally, Fig. $9 \mathrm{~b}$ shows that in the non-blocked time instants, the BR-Pre data rates slightly deteriorate compared to the BR-Det data rate by $4 \%$ and $3 \%$ at the median of the two speeds CDFs, and by $6 \%$ and $5 \%$ at the 25 -th percentile of the two speeds CDFs. These results show that during non-blocked time instants, the BRPre method performance difference to the BR-Det data rate, caused by the FP errors, are marginal.

In the remainder of this section, we take a deeper look at the data rate performance focusing on the lower part of the CDF presented in Fig. 9, as the worst served UEs are most likely to suffer in blocked time instants to meet the requirement of the most demanding applications. Focusing on the 25 th percentile results at blocker speed $1 \mathrm{~m} / \mathrm{s}$ shown in Fig. 10a, the BRDet and BR-Pre methods improve the BF data rate by $172 \%$ and $238 \%$ respectively and with BR-Pre outperforming BRDet by $24 \%$. Similarly, looking at 25 th percentile results of Fig. $10 \mathrm{~b}$ with the blocker moving at the speed of $2 \mathrm{~m} / \mathrm{s}$, the BR-Det and the BR-Pre methods improve the BF method data rate by $77 \%$ and $223 \%$, respectively. The advantage of BR-Pre over BR-Det increases, and it is assessed at $84 \%$. Note that the advantage offered by the BR-Pre method over the BR-Det one is more evident at the higher speed, as the reaction time of BR-Det does not scale with the higher frequency of the blockage events and their shorter duration, thus, by the time the method triggers the switch to the backup beam pair, the drop in the signal level caused by the blockage is either about to finish or already finished. In contrast, the BR-Pre data rate performance does not depend on the blocker speed, showing how predicting in advance the blockage occurrence is effective in indoor factory network deployments.

Similar considerations apply for the median and the 75th percentiles of CDFs with blocker speed of $2 \mathrm{~m} / \mathrm{s}$ showing $39 \%$ and $32 \%$ performance gains of our proposed prediction method over the detection one.

\section{CONCLUSION}

In this paper, we re-use existing beam measurement report messages from multiple UEs as input data for beam-specific DNN models that predict blockage events for multiple beams of the one exemplary BS. Our proposed multi-UE predictionbased method utilises the beam state predictions to control and trigger the beam recovery procedure ahead of the blockage events. This enables switching to a backup beam pair before the blockage disrupts the primary beam path. Thus, the prediction-based method allows for more stable signal quality and is more effective in significantly reducing the typical loss of data rate during the blockage event shown by commonly used methods based on detection. The validation of the proposed method with online predictions shows how data rate performance achieves $92 \%$ and $89 \%$ of the GT data rate for the blockers speeds of $1 \mathrm{~m} / \mathrm{s}$ and $2 \mathrm{~m} / \mathrm{s}$, respectively, confirming that the results are very close to the ideal case when the system has perfect knowledge of the future beam states. Moreover, the low number of FP errors rarely triggers the switching to the backup beam during non-blocked intervals and impact the data rate performance marginally, losing only 


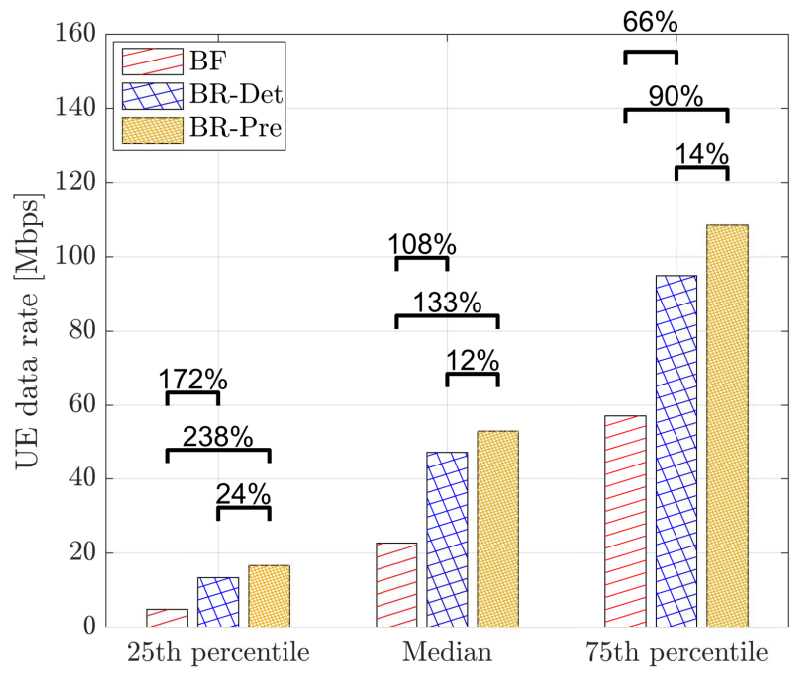

(a) UE data rate for blocker speed $1 \mathrm{~m} / \mathrm{s}$.

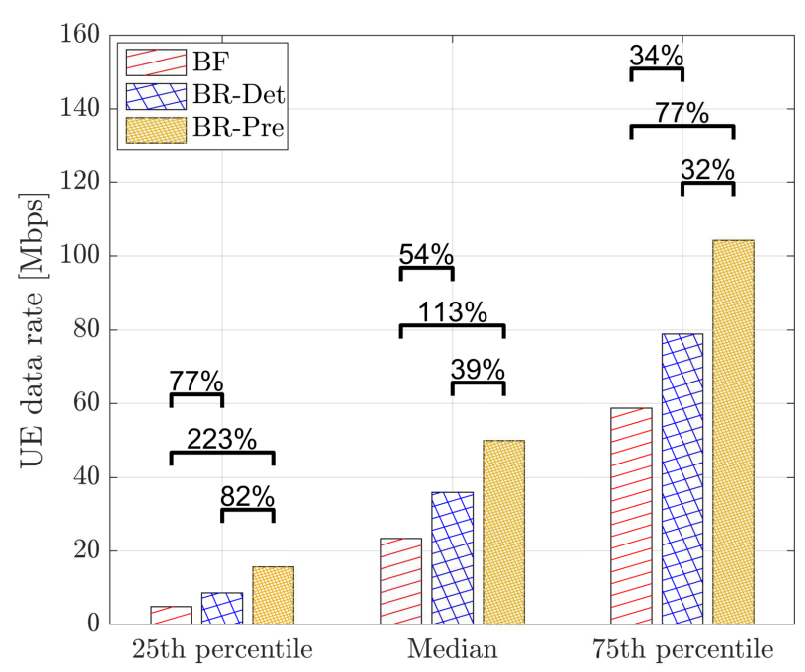

(b) UE data rate for blocker speed $2 \mathrm{~m} / \mathrm{s}$.

Fig. 10: 25-th, 50-th (median) and 75-th percentiles of the data rate performance for BR-Pre, BR-Det and BF methods during blocked time instants.

$4 \%$ and $3 \%$ to the BR-Det method median data rate. In addition, we show that the higher blocker speed penalises BRDet method as it is less effective in reacting to more frequent and shorter blockage events. In contrast, the BR-Pre data rate performance remains close to the GT. Finally, we found that for the worst served UE (at 25-th percentile of the CDF), the BR-Det improves the BF data rates by $172 \%$ and $77 \%$ for speeds $1 \mathrm{~m} / \mathrm{s}$ and $2 \mathrm{~m} / \mathrm{s}$, while BR-Pre improves BF method data rates by $238 \%$ and $223 \%$ for speeds $1 \mathrm{~m} / \mathrm{s}$ and $2 \mathrm{~m} / \mathrm{s}$. Overall, for the worst 25-th percentile UE, the proposed BRPre method improves the the BR-Det method data rates by $24 \%$ at a blocker speed of $1 \mathrm{~m} / \mathrm{s}$ and $82 \%$ at $2 \mathrm{~m} / \mathrm{s}$. Future works will focus on extending the current blockage scenario to diversify the blockage movement's trajectory and further reducing the time associated to the beam-specific training process.

\section{APPENDIX A \\ 3D Spatial Channel Model}

We generate the mmWave channel following the 3D SCM specified in [25], which considers a geometry-based stochastic channel model that accounts for a scattering environment formed by $N_{C}$ clusters individually composed by $N_{P}$ subpaths. The channel impulse response between the Tx-Rx antenna pair of the BS-UE link can be expressed as [25], [39], [40]

$$
\begin{aligned}
H_{u, s}(t, \tau)=\rho \sum_{\ell=1}^{N_{C}} \sum_{p=1}^{N_{P}} & \mathbf{F}_{R x}\left(\theta_{\ell, p}^{A}, \phi_{\ell, p}^{A}\right) e^{j \Phi_{\ell, p}} \mathbf{F}_{T x}\left(\theta_{\ell, p}^{D}, \phi_{\ell, p}^{D}\right) \\
& \times e^{j\left(\mathbf{k}_{R x, \ell, p}^{\top} \cdot \mathbf{d}_{R x, u}+\mathbf{k}_{T x, \ell, p}^{\top} \cdot \mathbf{d}_{T x, s}\right)} \\
& \times \sqrt{P_{\ell, p}} \cdot 10^{-\frac{B L_{\ell, p}(t)}{20}} \delta\left(\tau-\tau_{\ell, p}\right),
\end{aligned}
$$

where $\rho=\sqrt{10^{-\frac{P L+\sigma_{S F}}{10}}}$ represents the slow channel gain, which includes pathloss $P L$ and shadowing $\sigma_{S F}$. For each sub-path $p$ in cluster $\ell$, the model specifies $\operatorname{AoA}\left(\theta_{\ell, p}^{A}, \phi_{\ell, p}^{A}\right)$ and $\operatorname{AoD}\left(\theta_{\ell, p}^{D}, \phi_{\ell, p}^{D}\right)$, which modify the Rx and Tx antenna field patterns $\mathbf{F}_{R x}$ and $\mathbf{F}_{T x}$, and $\Phi_{\ell, p}$, which represents a random initial phase if polarisation is not considered. Moreover, the terms $\exp \left(j \mathbf{k}_{R x, \ell, p}^{\top} \cdot \mathbf{d}_{R x, u}\right)$ and $\exp \left(j \mathbf{k}_{T x, \ell, p}^{\top} \cdot \mathbf{d}_{T x, s}\right)$ represent the array responses of the Rx and Tx antennas, where $\mathbf{k}_{R x, \ell, p}$ and $\mathbf{k}_{T x, \ell, p}$ are the $\mathrm{Rx}$ and $\mathrm{Tx}$ wave vectors along the directions of the $p$-th sub-path in cluster $\ell$ such that $\|\mathbf{k}\|=\frac{2 \pi}{\lambda_{0}}$. Additionally, $\mathbf{d}_{R x, u}$ is the location vector of the receiving antenna $s$ whereas $\mathbf{d}_{T x, s}$ is that of the transmitting antenna $u$ computed in the global Cartesian coordinate system. Finally, for each sub-path $p$ in cluster $\ell$, the model accounts for the power gain $P_{\ell, p}$, blockage loss $B L_{\ell, p}(t)$ and propagation delay $\tau_{\ell, p}$. To note that Eq. (11) represents the channel impulse response for the NLoS case. Thus, we add the LoS channel coefficient to Eq. (11) and we scale both terms by the Rician $\mathrm{K}$-factor $K_{R}$. The resulting channel impulse response for the LoS case can be expressed as

$$
\begin{aligned}
H_{u, s}^{\mathrm{LoS}}(t, \tau) & =\sqrt{\frac{1}{K_{R}+1}} H_{u, s}^{\mathrm{NLoS}}(t, \tau) \\
& +\sqrt{\frac{K_{R}}{K_{R}+1}} H_{u, s, 1}^{\mathrm{LoS}}(t) \delta\left(\tau-\tau_{1}\right),
\end{aligned}
$$

where $H_{u, s, 1}^{\mathrm{LoS}}(t)$ and $\tau_{1}$ are the channel impulse response and the propagation delay of the LoS path, respectively [39].

\section{APPENDIX B \\ BLOCKAGE MODEL}

To capture the blockage loss given by blockers moving in the environment, we consider the 3GPP Blockage Model $\mathrm{B}$, which introduces a time-dependent component $B L(t)$ that 
TABLE II: 3GPP-based system-level simulation parameters

\begin{tabular}{|c|c|c|}
\hline \multirow{3}{*}{ NR Numerology } & Carrier frequency & $28 \mathrm{GHz}$ \\
\hline & System bandwidth / Total RBs & $396 \mathrm{MHz} / 275 \mathrm{RBs} \mid \overline{24}$ \\
\hline & Sub-carrier spacing / TTI duration & $120 \mathrm{KHz} / 0.125 \mathrm{~ms} \mid \overline{\overline{24}}$ \\
\hline \multirow{6}{*}{ BS description } & Network layout & Room size of $40 \mathrm{~m} \times 50 \mathrm{~m} \times 3 \mathrm{~m}, 4$ sites, 3 sectors/site \\
\hline & Deployment & Grid-based with ISD: $20 \mathrm{~m}$, height: $3 \mathrm{~m}$ \\
\hline & Antenna array & $\begin{array}{l}\text { UPA with element spacing } 0.5 \lambda \text {, Number of antennas per ar- } \\
\text { ray: } 8 \times 8=64 \text {, mechanical downtilt: } 20^{\circ} \text {, antenna boresigh } \\
\left.\left\{30^{\circ}, 150^{\circ}, 270^{\circ}\right\} \mid 26\right]\end{array}$ \\
\hline & Single antenna element pattern & $90^{\circ} \mathrm{H} \times 90^{\circ} \mathrm{V}$ beamwidths, $5 \mathrm{dBi} \max .26$ \\
\hline & Beamforming & Fully-analog architecture based on codebook with size $N_{\mathrm{CB}, T x}=64$ \\
\hline & Tx power & $20 \mathrm{dBm}$ \\
\hline \multirow{5}{*}{ UE description } & Deployment & Random, 20 UEs/sector on average, all UEs served, height: $1 \mathrm{~m}$ \\
\hline & Antenna array & $\begin{array}{l}\text { UPA with element spacing } 0.5 \lambda \text {, Number of antennas per array: } \\
\left.4 \times 4=16 \text {, antenna boresigh } \sim \mathcal{U}\left[0^{\circ}, 360^{\circ}\right] \mid 26\right]\end{array}$ \\
\hline & Single antenna element pattern & $90^{\circ} \mathrm{H} \times 90^{\circ} \mathrm{V}$ beamwidths, $5 \mathrm{dBi} \max$. \\
\hline & Beamforming & Fully-analog architecture based on codebook with size $N_{\mathrm{CB}, R x}=16$ \\
\hline & Noise figure & $10 \mathrm{~dB}$ \\
\hline \multirow{3}{*}{$\begin{array}{c}\text { Blocker } \\
\text { description }\end{array}$} & Model & Geometric-based model B 25 \\
\hline & Dimensions & $2 \mathrm{~m} \times 3 \mathrm{~m}$ \\
\hline & Trajectory & Linear with speed $v=\{1,2\} \mathrm{m} / \mathrm{s}$ \\
\hline \multirow{4}{*}{$\begin{array}{l}\text { mmWave } \\
\text { Channel } \\
\text { Description }\end{array}$} & Path loss and LOS probability & 3GPP 3D InH-Open office 25 \\
\hline & Shadowing & Log-normal with $\sigma=3 / 8 \mathrm{~dB}(\mathrm{LoS} / \mathrm{NLoS})$ \\
\hline & Fast fading & Ricean with log-normal K-factor $|\overline{25}|$ \\
\hline & Thermal noise & $-174 \mathrm{dBm} / \mathrm{Hz}$ power spectral density \\
\hline
\end{tabular}

reduces the power of the 3D channel cluster's sub-path [25]. The Blockage Model B deploys a 3D rectangular screen with sizes $w \times h$ for each blocker moving in the scenario and computes the total power loss given by the knife-edge diffraction from the edges of the screen [41]. The model maintains consistent results over time, space, and channel frequency components because of its geometric approach. The overall blockage attenuation can be expressed as [25]

$$
B L=-20 \log _{10}\left(1-\left(F_{h_{1}}+F_{h_{2}}\right)\left(F_{w_{1}}+F_{w_{2}}\right)\right),
$$

where $F_{h_{1}}, F_{h_{2}}, F_{w_{1}}, F_{w_{2}}$ represent the diffraction losses observed at the Rx corresponding to the four edges of the screen and can be expressed as [25]

$$
F_{w_{1,2} \mid h_{1,2}}=\left\{\begin{array}{l}
\frac{\tan ^{-1}\left( \pm \frac{\pi}{2} \sqrt{\frac{\pi}{\lambda_{0}}\left(D 1_{w_{1,2} \mid h_{1,2}}+D 2_{w_{1,2} \mid h_{1,2}}-d\right)}\right)}{\pi} \\
\text { for LoS path, } \\
\frac{\tan ^{-1}\left( \pm \frac{\pi}{2} \sqrt{\frac{\pi}{\lambda_{0}}\left(D 1_{w_{1,2} \mid h_{1,2}}-d^{\prime}\right)}\right)}{\pi} \\
\text { for NLoS path, }
\end{array}\right.
$$

where $\lambda_{0}$ is the wavelength, $d$ and $d^{\prime}$ are the BS-UE and blocker-UE distances, corresponding to the LoS and NLoS paths, respectively. $D 1_{w_{1,2} \mid h_{1,2}}$ and $D 2_{w_{1,2} \mid h_{1,2}}$ denote the line segments connecting the screen edges to the Tx or Rx points. These distances are evaluated in the top view for $w 1$ and $w 2$ and in the side view for $h 1$ and $h 2$. If the screen intersects the $\mathrm{Rx}$ path, the + sign is applied at both edges. Differently, if the screen does not intersect the Rx path and one of the edges is still diffracting the signal, the - sign is applied to the edge closest to the Rx path and the + sign is applied to the edge farthest from the Rx path.

\section{APPENDIX C \\ 3GPP-BASED SYSTEM-LEVEL SETUP}

Table III provides the set of simulation parameters used to configure the system-level simulator.

\section{REFERENCES}

[1] O. Simeone, "A very brief introduction to machine learning with applications to communication systems," IEEE Transactions on Cognitive Communications and Networking, vol. 4, no. 4, pp. 648-664, 2018.

[2] L. Gavrilovska, V. Atanasovski, I. Macaluso, and L. A. DaSilva, "Learning and reasoning in cognitive radio networks," IEEE Communications Surveys Tutorials, vol. 15, no. 4, pp. 1761-1777, 2013.

[3] Y. Sun, M. Peng, Y. Zhou, Y. Huang, and S. Mao, "Application of machine learning in wireless networks: Key techniques and open issues," IEEE Communications Surveys Tutorials, vol. 21, no. 4, pp. 3072-3108, 2019.

[4] S. Rangan, T. S. Rappaport, and E. Erkip, "Millimeter-wave cellular wireless networks: Potentials and challenges," Proceedings of the IEEE, vol. 102, no. 3, pp. 366-385, 2014.

[5] A. Ghosh, T. A. Thomas, M. C. Cudak, R. Ratasuk, P. Moorut, F. W. Vook, T. S. Rappaport, G. R. MacCartney, S. Sun, and S. Nie, "Millimeter-wave enhanced local area systems: A high-data-rate approach for future wireless networks," IEEE Journal on Selected Areas in Communications, vol. 32, no. 6, pp. 1152-1163, 2014.

[6] T. Bai and R. W. Heath, "Coverage and rate analysis for millimeter-wave cellular networks," IEEE Transactions on Wireless Communications, vol. 14, no. 2, pp. 1100-1114, 2015.

[7] J. Kibiłda et al., "Indoor millimeter-wave systems: Design and performance evaluation," Proceedings of the IEEE, vol. 108, no. 6, pp. 923944, 2020.

[8] S. Kutty and D. Sen, "Beamforming for millimeter wave communications: An inclusive survey," IEEE Communications Surveys \& Tutorials, vol. 18, no. 2, pp. 949-973, 2016.

[9] G. R. MacCartney, T. S. Rappaport, and S. Rangan, "Rapid fading due to human blockage in pedestrian crowds at $5 \mathrm{G}$ millimeterwave frequencies," in 2017 IEEE Global Communications Conference (GLOBECOM), 2017, pp. 1-7.

[10] C. Slezak, M. Zhang, M. Mezzavilla, and S. Rangan, "Understanding end-to-end effects of channel dynamics in millimeter wave $5 \mathrm{G}$ new radio," in 2018 IEEE 19th International Workshop on Signal Processing Advances in Wireless Communications (SPAWC), 2018, pp. 1-5. 
[11] R. Ford, M. Zhang, M. Mezzavilla, S. Dutta, S. Rangan, and M. Zorzi, "Achieving ultra-low latency in 5G millimeter wave cellular networks," IEEE Communications Magazine, vol. 55, no. 3, pp. 196-203, March 2017.

[12] 3GPP Technical Specification (TS) 38.321, "NR; Medium Access Control (MAC) protocol specification," Version 16.0.0, Apr. 2020.

[13] Y. M. Tsang and A. S. Y. Poon, "Detecting human blockage and device movement in mmWave communication system," in 2011 IEEE Global Telecommunications Conference (GLOBECOM) 2011, 2011, pp. 1-6.

[14] M. Jasim, M. Ababneh, N. Siasi, and N. Ghani, "Hybrid beamforming for link recovery in millimeter wave communications," in 2018 IEEE 19th Wireless and Microwave Technology Conference (WAMICON), 2018, pp. 1-4.

[15] S. H. A. Shah, M. Sharma, and S. Rangan, "LSTM-based multi-link prediction for mmwave and Sub-THz wireless systems," in ICC 2020 2020 IEEE International Conference on Communications (ICC), 2020, pp. $1-6$.

[16] M. Zarifneshat, L. Xiao, and J. Tang, "Learning-based blockage prediction for robust links in dynamic millimeter wave networks," in 2019 16th Annual IEEE International Conference on Sensing, Communication, and Networking (SECON), June 2019, pp. 1-9.

[17] M. Alrabeiah and A. Alkhateeb, "Deep learning for mmWave beam and blockage prediction using Sub-6 GHz channels," IEEE Transactions on Communications, vol. 68, no. 9, pp. 5504-5518, 2020.

[18] Y. Wang, A. Klautau, M. Ribero, A. C. K. Soong, and R. W. Heath "MmWave vehicular beam selection with situational awareness using machine learning," IEEE Access, vol. 7, pp. 87 479-87 493, 2019.

[19] T. Nishio, H. Okamoto, K. Nakashima, Y. Koda, K. Yamamoto, M. Morikura, Y. Asai, and R. Miyatake, "Proactive received power prediction using machine learning and depth images for mmWave networks," IEEE Journal on Selected Areas in Communications, vol. 37, no. 11, pp. 2413-2427, 2019.

[20] Y. Koda, K. Nakashima, K. Yamamoto, T. Nishio, and M. Morikura, "Handover management for mmWave networks with proactive performance prediction using camera images and deep reinforcement learning," IEEE Transactions on Cognitive Communications and Networking, vol. 6, no. 2, pp. 802-816, 2020.

[21] L. Simić, J. Arnold, M. Petrova, and P. Mähänen, "RadMAC: Radarenabled link obstruction avoidance for agile mm-wave beamsteering," in Proceedings of the 3rd Workshop on Hot Topics in Wireless, 2016, pp. 61-65.

[22] A. Alkhateeb, I. Beltagy, and S. Alex, "Machine learning for reliable mmWave systems: blockage prediction and proactive handoff," in 2018 IEEE Global Conference on Signal and Information Processing (GlobalSIP), 2018, pp. 1055-1059.

[23] Z. Ali, A. Duel-Hallen, and H. Hallen, "Early warning of mmWave signal blockage and AoA transition using sub-6 GHz observations," IEEE Communications Letters, vol. 24, no. 1, pp. 207-211, 2020.

[24] 3GPP Technical Specification (TS) 38.300, "NR; Overall description; Stage-2," Version 16.1.0, Apr. 2020.

[25] 3GPP Technical Report (TR) 38.901, "Study on channel model for frequencies from 0.5 to $100 \mathrm{GHz}$," Version 16.1.0, Jan. 2020.

[26] 3GPP RT-170019, "Summary of Email Discussion [ITU-R AH 01] Calibration for Self-Evaluation," 3GPP TSG RAN ITU-R ad hoc, Dec. 2017.

[27] 3GPP Technical Specification (TS) 38.211, "NR; Physical channels and modulation," Version 16.1.0, Apr. 2020.

[28] 3GPP Technical Report (TR) 38.802, "Study on new radio access technology physical layer aspects," Version 14.2.0, Sep. 2017.

[29] 3GPP R1-1802446, "Discussion on the RSRP calculation," China Telecom, Feb. 2018

[30] 3GPP Technical Specification (TS) 38.213, "NR; Physical layer procedures for control," Version 16.1.0, Apr. 2020.

[31] M. Giordani, M. Polese, A. Roy, D. Castor, and M. Zorzi, "A tutorial on beam management for 3GPP NR at mmWave frequencies," IEEE Communications Surveys \& Tutorials, vol. 21, no. 1, pp. 173-196, Firstquarter 2019.

[32] K. He, X. Zhang, S. Ren, and J. Sun, "Delving deep into rectifiers: Surpassing human-level performance on imagenet classification," in The IEEE International Conference on Computer Vision (ICCV), Dec. 2015.

[33] A. F. Agarap, "Deep Learning using Rectified Linear Units (ReLU)," arXiv e-prints, p. arXiv:1803.08375, Mar 2018.

[34] D. P. Kingma and J. Ba, "Adam: A Method for Stochastic Optimization," arXiv e-prints, p. arXiv:1412.6980, Dec 2014.
[35] H. He and E. A. Garcia, "Learning from imbalanced data," IEEE Transactions on Knowledge and Data Engineering, vol. 21, no. 9, pp. 1263-1284, Sep. 2009.

[36] C. M. Bishop, Pattern Recognition and Machine Learning. Berlin, Heidelberg: Springer, 2006.

[37] W. Yang and K. Liu, "Blockage effect and beam cooperation in indoor hotspot based on 3GPP NR blockage model," in ICC 2019 - 2019 IEEE International Conference on Communications (ICC), May 2019, pp. 1-

[38] M. Tayyab, X. Gelabert, and R. Jäntti, "A survey on handover management: From LTE to NR," IEEE Access, vol. 7, pp. 118907-118930, 2019.

[39] Q. Nadeem, A. Kammoun, and M. Alouini, "Elevation beamforming with full dimension MIMO architectures in 5G systems: A tutorial," IEEE Communications Surveys \& Tutorials, vol. 21, no. 4, pp. 32383273, 2019.

[40] A. Kammoun, H. Khanfir, Z. Altman, M. Debbah, and M. Kamoun, "Preliminary results on 3D channel modeling: From theory to standardization," IEEE Journal on Selected Areas in Communications, vol. 32, no. 6, pp. 1219-1229, 2014.

[41] J. Kunisch and J. Pamp, "Ultra-wideband double vertical knife-edge model for obstruction of a ray by a person," in 2008 IEEE International Conference on Ultra-Wideband, vol. 2, Sep. 2008, pp. 17-20. 